\title{
Mechanisms of interdecadal climate variability and the role of ocean-atmosphere coupling
}

\author{
Riccardo Farneti · Geoffrey K. Vallis
}

Received: 18 May 2009/ Accepted: 21 September 2009

(C) Springer-Verlag 2009

\begin{abstract}
Climate variability and mid-latitude mechanisms of ocean-atmosphere interactions are investigated with coupled and uncoupled integrations of a threedimensional ocean-atmosphere-land-ice climate model of intermediate complexity. We focus on the decadal and interdecadal variability of the system and give a statistical and dynamical description of its oceanic and atmospheric signatures. In our coupled control integration, an oceanic oscillation of a period of around 20 years is found to be associated with variability of the meridional overturning circulation and is manifested by surface anomalies of temperature and salinity. On such timescales the oceanic oscillation is able to imprint itself on the atmosphere, which then covaries with the ocean at the oscillation period. The essentially slaved atmospheric pattern helps maintain the oceanic oscillation by providing large-scale anomalous heat fluxes, so catalyzing the oscillation. That is to say, because the atmosphere covaries with the ocean the damping felt by the ocean is less than what would be felt with a fixed atmosphere, so broadening the parameter regime over which such variability occurs. In addition to the presence of an atmosphere, the period and amplitude of the oscillation are found to be influenced both by the oceanic vertical diffusivity $\kappa_{v}$, by geometrical factors, and by the presence of stochastic heat fluxes. In general, oscillations occur most readily for large values of $\kappa_{v}$, when the mean state of the ocean is characterized by a strong meridional overturning circulation. If $\kappa_{v}$ is sufficiently strong, the ocean will oscillate even in the absence of a
\end{abstract}

R. Farneti $(\bowtie) \cdot$ G. K. Vallis

GFDL/AOS Program, Princeton University, Princeton, NJ 08544, USA

e-mail: riccardo.farneti@noaa.gov dynamical atmosphere. However, for more realistic values of $\kappa_{v}$, the presence of an interacting atmosphere is required for significant oscillations. If the ocean is forced by imposed stochastic heat fluxes, instead of a fully interacting atmosphere, then decadal-scale oscillations can be produced suggestive of a damped oscillator. However, the parameter range over which oscillations occur is smaller than when the ocean is coupled to full atmosphere. More generically, the ability of comprehensive coupled oceanatmosphere models to produce multi-decadal variability, realistic or otherwise, will depend on the oceanic mean state, and so on the diapycnal diffusivity of the modelled ocean, as well as on the ability of the atmosphere to reduce the damping felt by the ocean and so on the atmosphere's ability to respond to persistent sea-surface temperature anomalies.

Keywords Interdecadal climate variability . Ocean-atmosphere coupling · Air-sea interaction . Atlantic meridional overturning circulation .

Atmospheric teleconnection patterns

\section{Introduction}

Understanding natural variability on interannual, decadal to centennial timescales is an important and longstanding problem in climate dynamics. The expected acceleration of anthropogenic warming makes understanding such variability even more of a priority, because it is important to distinguish such warming from natural variability. Such variability almost certainly involves air-sea interactions and both media are likely important. In this study we will be focusing on the decadal and longer variability of the extratropical ocean-atmosphere system, using an idealized 
model that has similarities to the Atlantic region with the aim of isolating some of the essential dynamics.

The most realistic tools available for this kind of study are state-of-the-art, 'IPCC-class' coupled general circulation models (CGCMs); however, these models also carry an extensive computational burden and the identification of a particular mechanism or cause-and-effect pathway in the climate system becomes difficult to discern. At the other extreme, one may construct very idealized models that allow for several century-long integrations, ensemble runs and parameter dependency studies. However, if the models are oversimplified their relevance to the real climate system becomes at issue as they may neglect crucial dynamics important to coupled variability, so that care must be taken in their interpretation and use.

In the past, many idealised coupled models for studies of interdecadal and longer timescales have omitted a fully dynamical atmospheric component, focusing on different degrees of simplification (from an Energy Balance Model (EBM) to statistical-dynamical models; e.g., Saravanan and McWilliams 1995; Kamenkovich et al. 2002; Kravtsov and Ghil 2004; Montoya et al. 2005), while other studies have opted for a very simplified physical framework via the use of quasi-geostrophic (QG) dynamics (e.g., Hogg et al. 2006).

In this paper we use an idealised climate model (Farneti and Vallis 2009) that retains the full three-dimensional dynamics in its ocean-atmosphere-ice components, but that makes simplifications in two ways: (1) We use simplified physical parameterisations, especially in the atmospheric component; for example, we use simplified radiation and convection schemes, and (2) We make simplifications in the geometry and geography, integrating only over a sector of the Earth and with a single (but crosshemisphere) ocean basin.

Given such simplifications, one cannot expect to reproduce an accurate climate in all its details. However, although highly idealised in its geometrical setting and atmospherical physical parameterisations, the model produces a fairly realistic climate and circulation in both the atmosphere and ocean representative of Atlantic presentday conditions. Our goal is then to identify mechanisms of low-frequency interactions in the coupled ocean-atmosphere system and try to understand the relative role of its sub-components under different configurations and couplings. Such mechanisms can then be later tested against results from more comprehensive models and, ideally, with observations.

When dealing with decadal and longer climate variability it is rather natural to focus on the ocean, for here is where the memory of the system likely resides because of its relatively huge heat capacity and slow dynamics. Nevertheless, some studies have found evidence for dynamically coupled modes of decadal variability (e.g., Latif and Barnett 1994; Timmermann et al. 1998). This prompts us to categorise air-sea interactions that take place at midlatitude and at decadal and longer timescales into the following groups (see for example Saravanan et al. 2000):

- In the first case, the atmosphere varies essentially as white noise and the ocean, of much higher heat capacity, passively integrates this variability (Hasselmann 1976).

- The second type of mechanism is that in which the ocean varies on interannual to interdecadal time scales; the oceanic variability is then imprinted onto an atmosphere that is partially 'slaved' to the ocean at those periods, although it can and will provide additional, independent, variability at shorter time scales. The cause of the oceanic variations might be purely internal to the ocean, stemming from a hydrodynamic instability (Colin de Verdière and Huck 1999; Huck and Vallis 2001).

- In a variant of this scenario, a third case is characterised by the ocean acting as a damped oscillator that requires some excitation or stochastic noise of some sort, and this could be provided by atmospheric variability on much shorter timescales than the oscillation itself (Griffes and Tziperman 1995; Delworth and Greatbatch 2000). In neither case is the nature of the stochastic forcing significantly modified by the oceanic oscillations, and so the mode cannot properly be said to be a coupled one.

- A distinct fourth mechanism involves non-trivial feedbacks between the atmosphere and ocean giving rise to unstable or weakly damped coupled modes of the system, such as are generally believed to be involved in El Niño and the Southern Oscillation. In midlatitudes such a mechanism was identified by Latif and Barnett (1994) but has not been robustly reproduced.

A number of prior studies using fairly complete CGCMs have investigated the natural variability of the Atlantic ocean on decadal timescales, (e.g., Delworth et al. 1993; Timmermann et al. 1998; Dong and Sutton 2005; Zhu and Jungclaus 2008; Danabasoglu 2008). In spite of all this effort, little consensus has been reached so far on the underlying mechanism and cause of the variability, period of oscillation and strength of the air-sea coupling. Whilst observational estimates point to a variability in SSTs of around 40-70 years from proxy data (Kushnir 1994; Delworth and Mann 2000), model results span a variety of periods of oscillation and, most importantly, different sensitivity of the middle troposphere to SST signals and thus coupling between the ocean and atmosphere at the decadal time scale. We will argue that the ocean model formulation, and consequently its mean state, have a crucial role in setting the variability of the ocean and its ability 
to drive the atmosphere at decadal and longer timescales. When this mechanism is active, it can reinforce the oceanic oscillation through a reduced thermal damping of the SST anomalies via surface fluxes, as postulated by Barsugli and Battisti (1998).

An objective of the present study is therefore to analyse a computationally efficient coupled model of intermediate complexity that serves as a bridge between uncoupled or highly idealized models and CGCMs. We briefly describe the model in Sect. 2. In Sect. 3 the variability of the system is analysed and its dependence on the vertical diffusivity and dimensions of the ocean is given in Sect. 4. The hypotheses proposed for the interdecadal variability, the role of the ocean mean state and coupling with the atmosphere are confirmed in Sect. 5 with several targeted uncoupled simulations of the same model. We conclude with a summary of the main results and their discussion in Sect. 6.

\section{The model}

The model used in this study is a coupled atmosphereocean-ice-land model directly derived from the Geophysical Fluid Dynamics Laboratory (GFDL) Coupled Model 2.0. However, the physical parameterizations of the atmosphere and the general geometry and geography of the model are simplified in comparison with a more comprehensive climate model. An extensive description of the model components and configurations is given in Farneti and Vallis (2009) and thus our discussion here will be brief.

The atmospheric model is a moist, hydrostatic primitive equation model, using idealised physical parameterisations (Frierson et al. 2006, 2007; Frierson 2007) and configured as a $120^{\circ}$ re-entrant sector. The atmosphere is based on the GFDL 'B-grid' dynamical core with a horizontal resolution of $3.75^{\circ} \times 3^{\circ}$ extending between $75^{\circ} \mathrm{N}$ and $\mathrm{S}$ and seven vertical levels. The model utilises a simplified Betts-Miller convective scheme and Monin-Obukhov boundary layer. Infra-red radiation is calculated using a grey scheme with fixed emissivities, so that the upward and downward fluxes are functions of temperature only, and the atmosphere is taken to be transparent to solar radiation, which has neither a diurnal nor an annual cycle.

The ocean model is MOM4 (Griffes et al. 2005), which is a free-surface primitive equation model in $z$-coordinates. The main simplification is that there is a single basin, which in our control configuration is $60^{\circ}$ wide, extending from $70^{\circ} \mathrm{S}$ to $70^{\circ} \mathrm{N}$, representative of the Atlantic basin. In the region from $65^{\circ} \mathrm{S}$ to $51^{\circ} \mathrm{S}$ the walls are removed and the basin is periodic in longitude, thus recreating the topology of the Southern Ocean. The horizontal resolution is $2^{\circ}$, there are 24 levels, and the ocean is flat-bottomed with a depth of 4000m, except in the model Drake Passage where the total depth is reduced to $2,500 \mathrm{~m}$. In the Control (CTL) experiment, the model uses a constant diapycnal diffusion $\left(5 \times 10^{-5} \mathrm{~m}^{2} \mathrm{~s}^{-1}\right)$ and a Gent-McWilliams-Redi type scheme for parameterising mesoscale eddies, which is set to $0.8 \times 10^{3} \mathrm{~m}^{2} \mathrm{~s}^{-1}$. The ocean is coupled to the GFDL dynamical-thermodynamical sea-ice model (Hunke and Dukowicz 1997; Winton 2000) at the same horizontal resolution. Finally, a land model is implemented as a single bucket land, with constant values of water availability, heat capacity, roughness and drag coefficients. Rivers redistribute the precipitated water back into the ocean at the nearest grid point.

Overall, the idealised coupled model is capable of reproducing a fairly realistic Atlantic-like climate in both the ocean and atmosphere and, due to its computational efficiency, it allows integrations of several millennia as well as broad parameter studies (Farneti and Vallis 2009).

\section{Interdecadal variability}

\subsection{Oceanic variability}

Annually averaged data for both the ocean and atmosphere are used in the following analysis. Since our interest is focused on decadal-interdecadal climate variability, we make use of a 5-year low-pass Lanzcos filter in time applied to our variables, unless otherwise stated. Figure 1 shows the time-mean meridional overturning circulation (MOC) and the annual anomalies of the MOC time series, computed as the maximum value in the box $\left[50^{\circ} \mathrm{N}-70^{\circ} \mathrm{N}\right.$; $0-2,500 \mathrm{~m}$ ] during the coupled integration. Quasi-regular oscillations of interdecadal period are evident throughout the millennial run and their averaged amplitude is about $2 \mathrm{~Sv}\left(1 \mathrm{~Sv} \equiv 10^{6} \mathrm{~m}^{3} \mathrm{~s}^{-1}\right)$, or $\sim 20 \%$, of the time mean transport.

Oscillations are also present in a time series of the Northern Hemisphere SST anomalies (computed as the detrended weighted average north of $50^{\circ} \mathrm{N}$ ) and are shown to share the MOC spectral peak at around 20 years in Fig. 2. Here, the Fourier spectral power of the two indices are plotted and tested against a first-order autoregressive process $\mathrm{AR}(1)$ and the 95\% confidence level for the rejection of the red noise hypothesis. The interdecadal peak is highly significant in both fields, while at shorter periods the signal follows a red noise prediction, and at longer periods the spectra flatten out.

Our results show an interdecadal mode that is evidently more than just the integration of some atmospheric white noise, although inspection of time series or power spectra cannot identify the origin or mechanism of the oscillation. To proceed, we first describe the oceanic variability in 
Fig. 1 (top panel) Time mean meridional overturning circulation (c.i. $=2 \mathrm{~Sv}$ ) for the CTL experiment. The deep cell in the North of the basin peaks at about $12 \mathrm{~Sv}$. (bottom panel) Annual anomalies of the MOC time series, computed as the maximum value in the box $\left[50^{\circ} \mathrm{N}-70^{\circ} \mathrm{N} ; 0-2500 \mathrm{~m}\right]$, show interdecadal variability of about $20 \%$ in amplitude
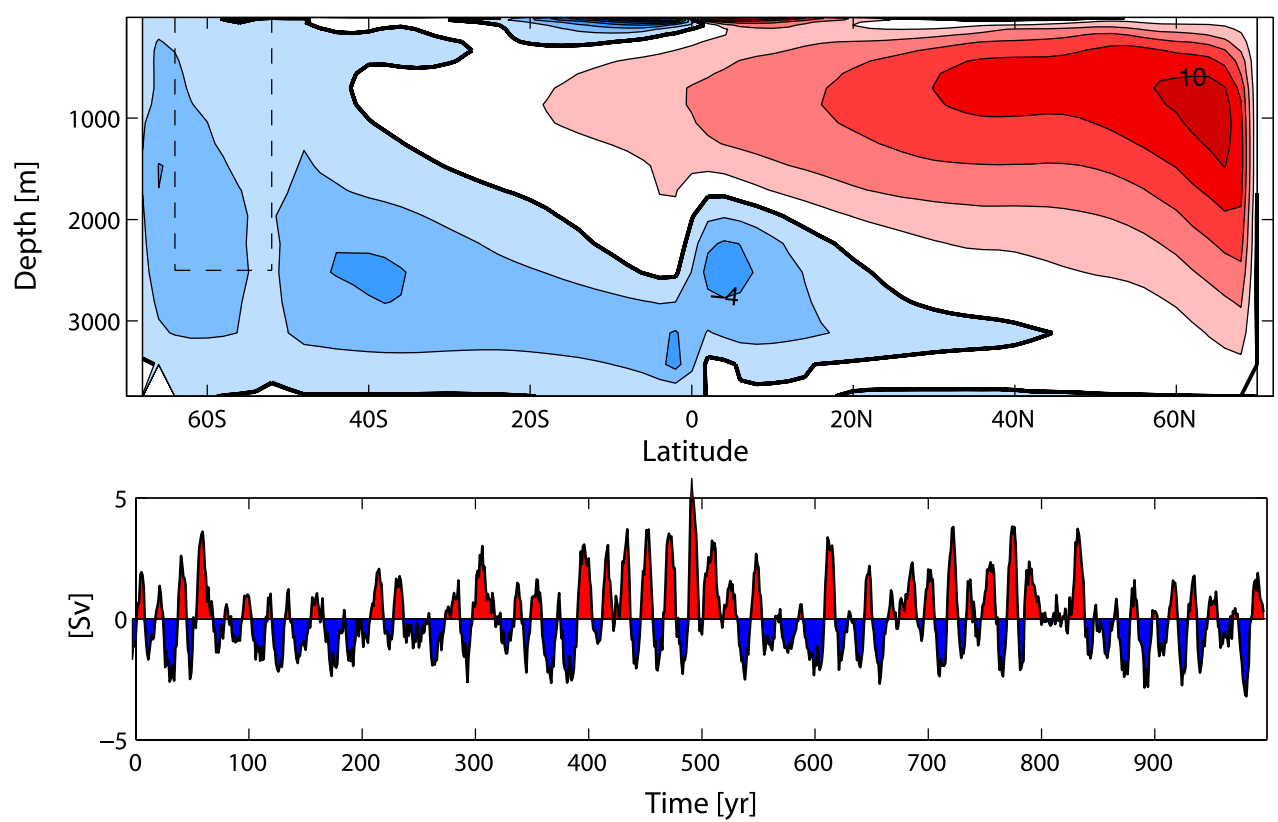

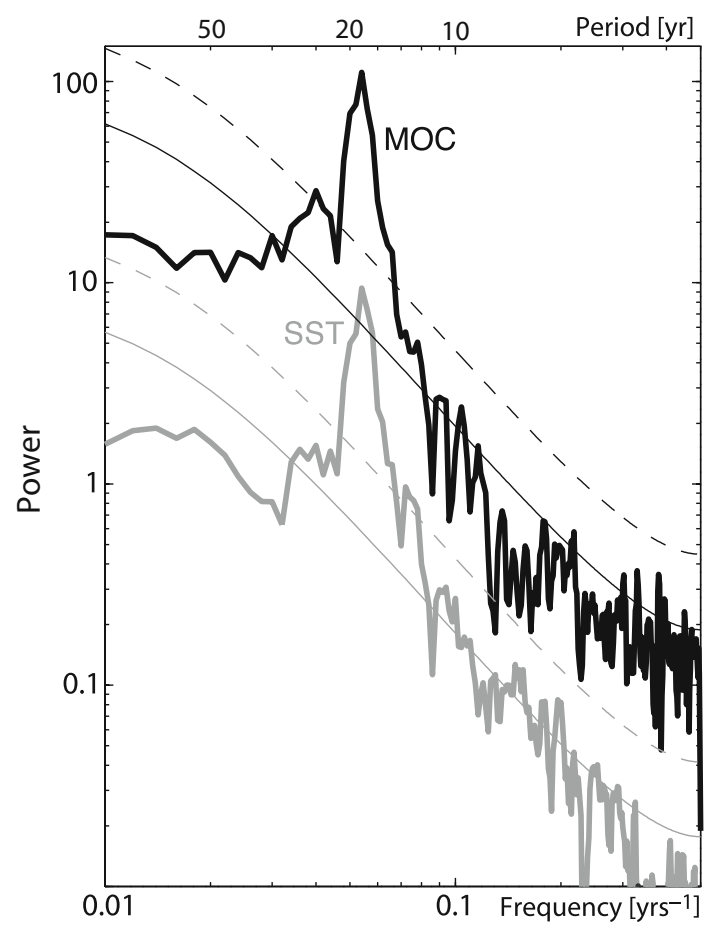

Fig. 2 Power spectra of the unfiltered SST $\left({ }^{\circ} \mathrm{C}^{2}\right.$ years) and MOC $\left(\mathrm{Sv}^{2}\right.$ years $)$ time series for the CTL experiment. The thin lines indicate their equivalent $\mathrm{AR}(1)$ processes and the dotted lines the red noise $95 \%$ confidence level. There is a high concentration of variance at the 20 years period for both variables and a red noise behaviour at higher frequencies

terms of Empirical Orthogonal Functions (EOFs). The first and second primary pattern of variability for MOC, SST and sea surface salinity (SSS) are shown in Fig. 3. An increase in strength in the meridional circulation of about $2 \mathrm{~Sv}$ is related to a dipolar-tripolar pattern of cold and fresh anomalies in the subpolar ocean $\left(50^{\circ} \mathrm{N}-70^{\circ} \mathrm{N}\right.$; top panels of Fig. 3). The meridional circulation and surface tracers share the same 20 years spectral peak (bottom panels of Fig. 3). The propagation of buoyancy anomalies from the western boundary to the region of convection (north eastern corner of the basin), depicted by the first two EOF spatial patterns, is demonstrated by the lagged correlation of the SST and SSS principal components (PCs) with respect to the MOC first PC (MOC index; $\Psi_{i}$ ). The first and second PC are highly correlated with $\Psi_{i}$ and in quadrature with each other, thus corresponding to a propagating pair covarying with a characteristic oscillation of about 20 years given by the $\Psi_{i}$ index (not shown).

Advection of tracer anomalies along the mean circulation and across mean temperature gradients has been previously found in a number of models and believed to stem from anomalies in the gyre or thermohaline circulation. Here, as in Saravanan et al. (2000), the anomalies are initiated by perturbation advection near the western boundary current and are then advected by the mean flow $\left(\bar{U} T^{\prime}\right)$ reaching the region of convection after a decade (Fig. 4); in contrast to an anomalous gyre advecting the mean tracer $\left(U^{\prime} \bar{T}\right)$ across the gyre boundary, as in Marshall et al. (2001). The oceanic mode resembles the one found by more comprehensive coupled models (Delworth et al. 1993; Timmermann et al. 1998; Dong and Sutton 2005; Dai et al. 2005, to cite a few), where the oscillation arises from the interplay between the thermal $\left(\rho_{\mathrm{s}}^{\prime}\right)$ and saline $\left(\rho_{\mathrm{t}}^{\prime}\right)$ contribution to the density anomalies $\rho^{\prime}$, as in a classical thermohaline oscillation. In Fig. 5 the thermal anomaly dominates-note how $\rho^{\prime}$ closely follows $\rho_{\mathrm{t}}^{\prime}$-leading the MOC to oscillate. Figure 5 also shows the MOC regressed onto its own index $\Psi_{i}$ (grey line), revealing a weakly damped oscillation. 

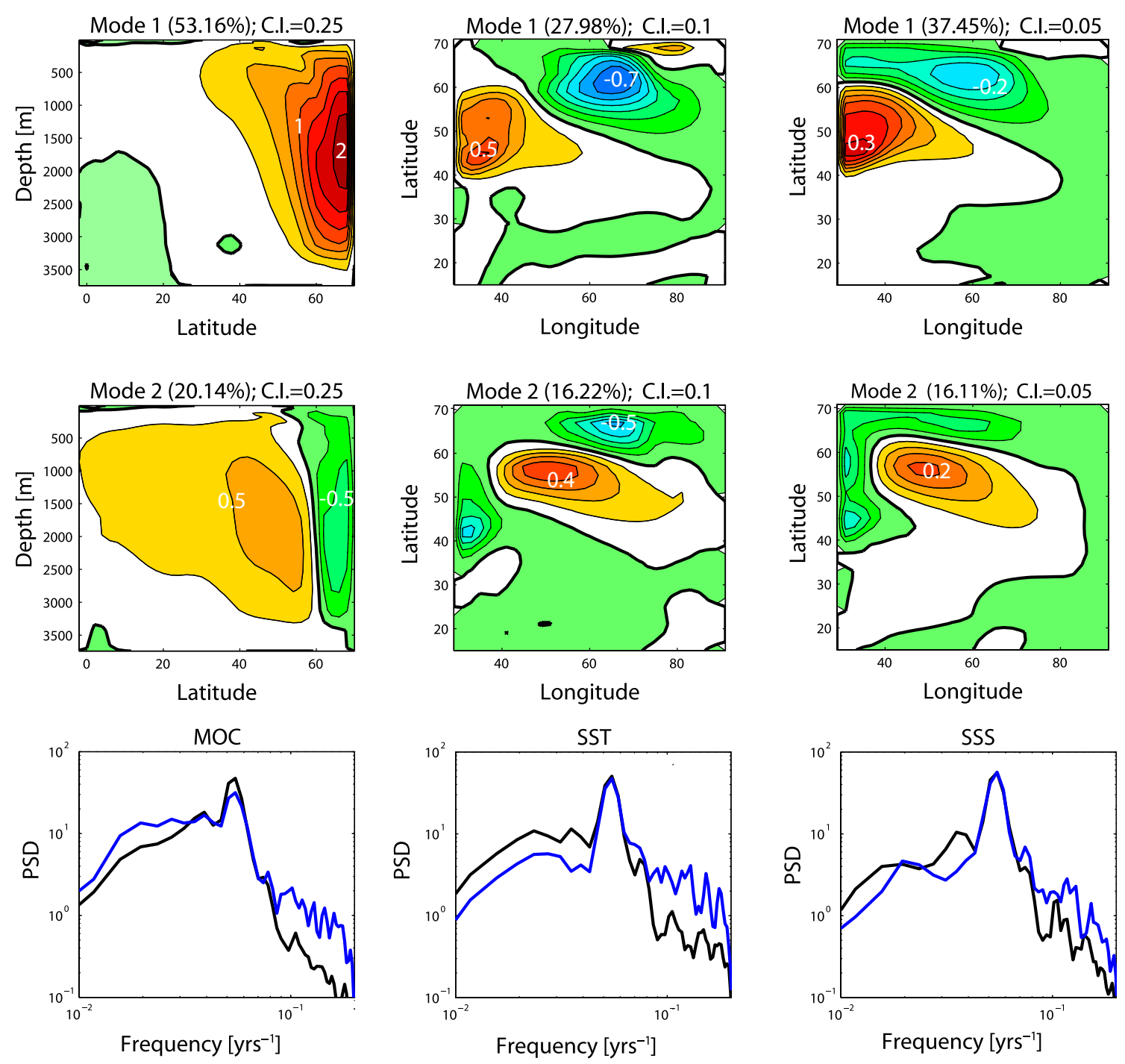

Fig. 3 First and second EOF spatial patterns for the MOC in Sv (left panels), SST in ${ }^{\circ} \mathrm{C}$ (centre panels) and SSS in psu (right panels). The percentage of variance explained and contour interval is at the top of

Coherent thermal and saline anomalies of decadal time scale propagating through the North Atlantic have been observed and modelled with models of various complexity (Deser and Blackmon 1993; Kushnir 1994; Sutton and Allen 1997), and may be part of an advective timescale of $\mathcal{O}$ (10 years) that is typical for the basin and mean flow of the subpolar North Atlantic. How the MOC instability is started and whether it is self-sustained, forced by stochastic atmospheric forcing or even part of a coupled feedback will be discussed in the following sections. Next, we will focus on the atmospheric variability and its possible response to these propagating oceanic anomalies. A fundamental question is whether we can unequivocally determine if some atmospheric variability is associated with, and possibly forced by, the oceanic variability. If so, what are the patterns associated with it? Such variability will of course each spatial pattern. The 5 year low-passed power spectral density shown at the bottom are for the first (black) and second (blue) normalized principal components

depend on the model's ability to respond to SST anomalies, and the strength of these anomalies.

\subsection{Atmospheric variability}

Internal atmospheric low-frequency variability in mid-latitudes is characterized by equivalent barotropic oscillations in the zonal wind, most noticeable in the regions of the storm tracks. In the Northern Hemisphere this is the "North Atlantic Oscillation' (NAO) and its projection on the zonally averaged flow is sometimes called the 'Northern Annular Mode'. The mechanism is internal to the atmosphere, and ultimately comes from baroclinic instability (Vallis and Gerber 2008), but this alone cannot produce significant interannual variability. We see below that decadal scale variability produced by our model has a 
Fig. 4 Time-longitude diagram of SST (left) and SSS (right) at latitude $55^{\circ} \mathrm{N}$ for a sample interval between model years 850 and 950 . The latitude was chosen as the region of maximum variance for both SST and SSS, other than the western boundary and the region of convection

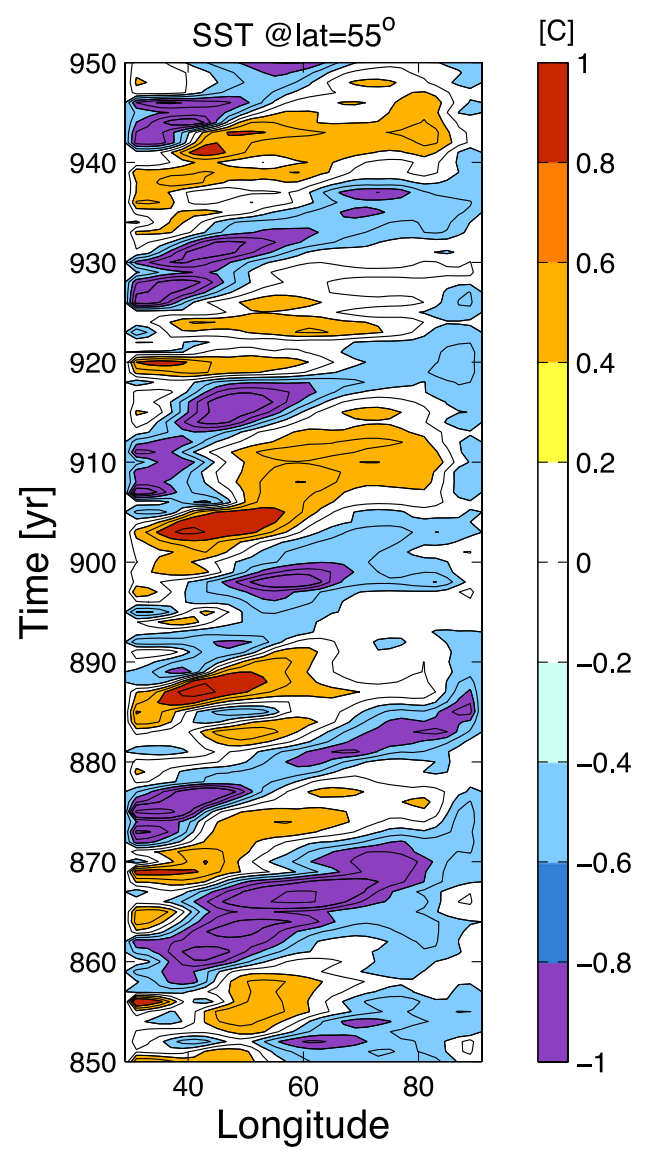

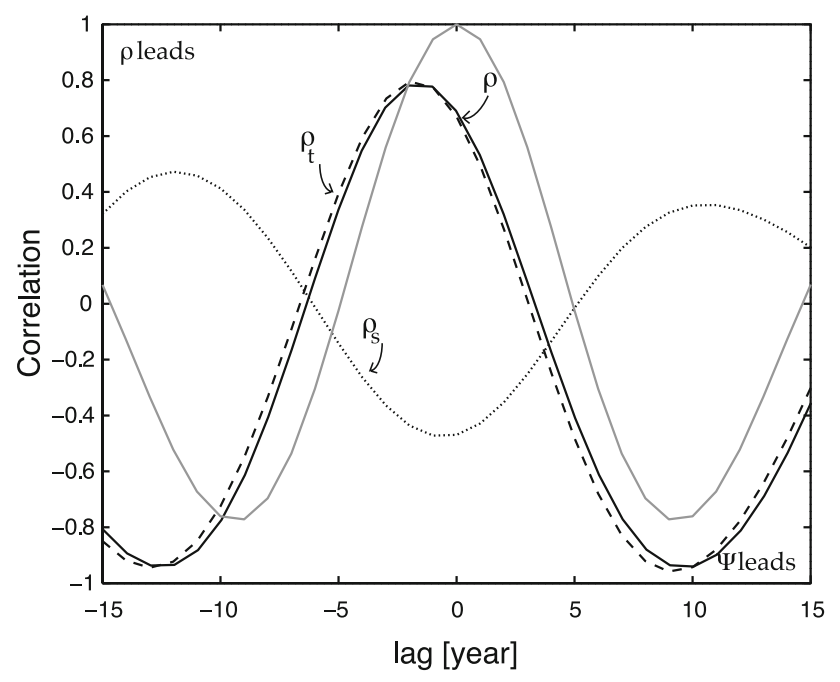

Fig. 5 Lagged correlation coefficients for the thermal $\left(\rho_{t}\right)$, saline $\left(\rho_{s}\right)$ and total $(\rho)$ horizontal density anomaly averaged in the subpolar area $\left[50^{\circ} \mathrm{N}-70^{\circ} \mathrm{N}\right]$ with respect to the MOC index $\Psi_{i}$. The correlation of $\Psi_{i}$ with itself is also plotted in grey for reference of the oscillation

similar structure to this shorter term variability. That is, the forcing arising from variations in the SST causes long-term variability with a similar structure as the natural patterns of variability of the atmosphere.
We first performed an EOF analysis of the 5 year low-passed atmospheric circulation and found that the low-frequency atmospheric variability is characterised by spatially coherent patterns of sea level pressure (SLP), in particular by two meridional dipoles capturing 45 and $39 \%$ of the total variance, respectively (Fig. 6). Although the idealised geometry and absence of orography in the atmospheric sector make a comparison with CGCMs and observational data rather difficult, we can identify the second mode of variability (i.e., the second EOF of the sealevel pressure) as the model's NAO. Because our midlatitude jet lies in the latitude $40^{\circ} \mathrm{N}-45^{\circ} \mathrm{N}$, rather equatorward of the observed climatology, it is the second mode of SLP that represents its modulation in strength. The eddy kinetic energy at $250 \mathrm{hPa}$, showing maxima at $40^{\circ} \mathrm{N}-50^{\circ} \mathrm{N}$ (i.e., our storm track) confirms this conclusion. Figure 6 also shows the spectra of the annual-mean principal components of the first and second SLP EOF spatial patterns. The spectra are essentially white at interannual periods but a narrow interdecadal peak in EOF-2, whose 20 year time scale is the same as the one found in the ocean, is well above the $95 \%$ confidence interval for the reference red noise spectrum. Also, the interdecadal SLP dipolar pattern has an equivalent-barotropic structure as indicated by an 

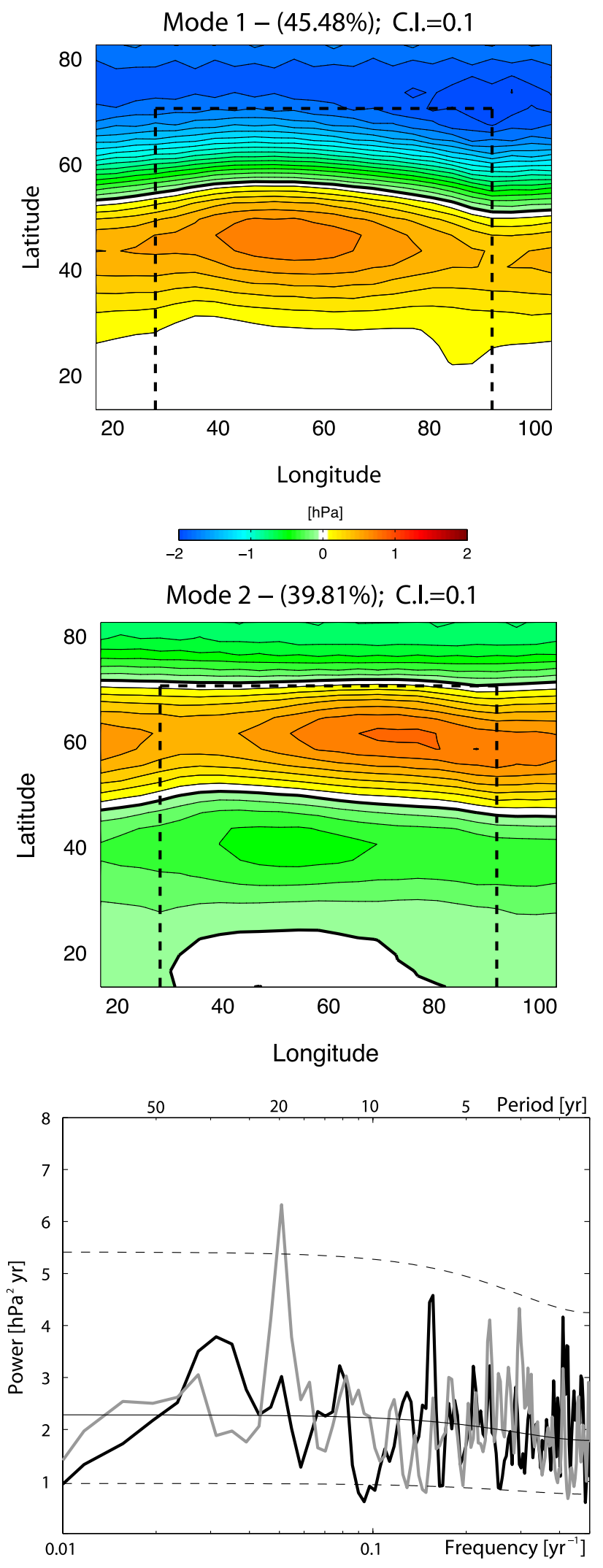

Fig. 6 Spatial patterns of EOF-1 and 2 of SLP (hPa). Both modes capture a large fraction of the total variance $(\sim 45$ and $\sim 40 \%)$. The contour interval is $0.1 \mathrm{hPa}$ and the dashed box represents the ocean basin extent. First (black) and second (grey) spectral density of the low-passed SLP EOFs principal components. The thin solid line denotes the spectrum of an equivalent red noise process and the dashed lines are the 5 and $95 \%$ confidence level for accepting the red noise null hypothesis

EOF on the geopotential height anomalies at 880,450 and $250 \mathrm{mb}$ (not shown).

The nature of low frequency atmospheric power, and whether it is a consequence of oceanic forcing, will be studied later on with uncoupled experiments, where the influence of the atmosphere on the ocean and vice-versa will be explored in detail under different forcings. Prior to that we will try to understand the evolution of the atmospheric mode and its possible relationship to the oceanic conditions during an MOC cycle.

\subsection{Coupled variability}

The relationship between oceanic and atmospheric fields is studied by means of a lagged regression analysis of SST, SSS and SLP onto the MOC index $\Psi_{i}$, as shown in Fig. 7. When the MOC is at its maximum (lag 0 year) strong negative SST anomalies are found in the upper subpolar region together with negative SSS anomalies. This situation tends to strengthen the meridional temperature gradient in the atmosphere and supports a positive phase of our SLP EOF-2 (anomalously low (high) in the upper (lower) centre of action; upper right panel of Fig. 7). Because of the vigorous overturning circulation, the subpolar basin fills up with warm salty water (lag +4 years, centre panels) generated by the anomalous heat transport convergence at the region of maximum temperature gradient. Anomalies are later advected by the mean flow from the western boundary towards the region of convection (Fig. 4). At this stage, the atmospheric dipole is still on its positive phase but somewhat weakened. At lag +9 years (bottom panels), i.e. $\Psi_{i}$ is at its minimum, warm and salty waters have reached the northern boundary of the basin, precluding convection (density anomalies are temperature dominated, Fig. 5) and reverting the phase in the SLP dipole. Now the oscillation enters its negative phase, caused by the oceanic influence of anomalous warm waters and, it is supposed, positive atmospheric heat fluxes, with SST and SLP covarying during the interdecadal oscillation.

It is possible, of course, that the atmospheric dipole pattern could be the trigger and not the response of the 

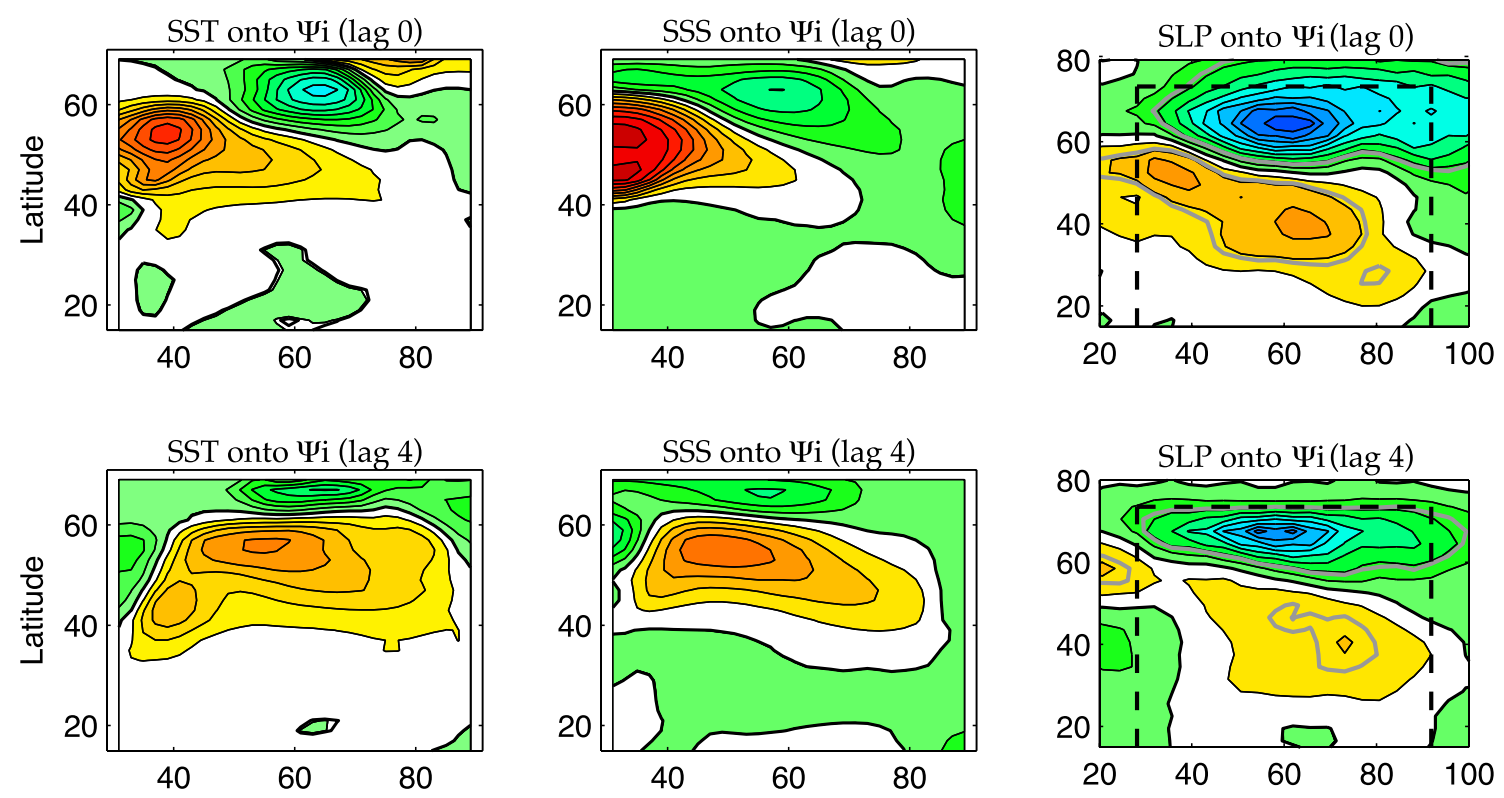

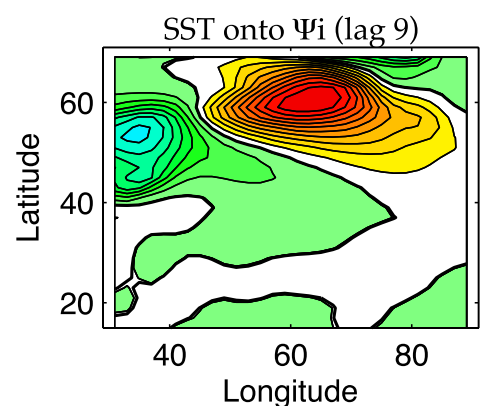

[C]
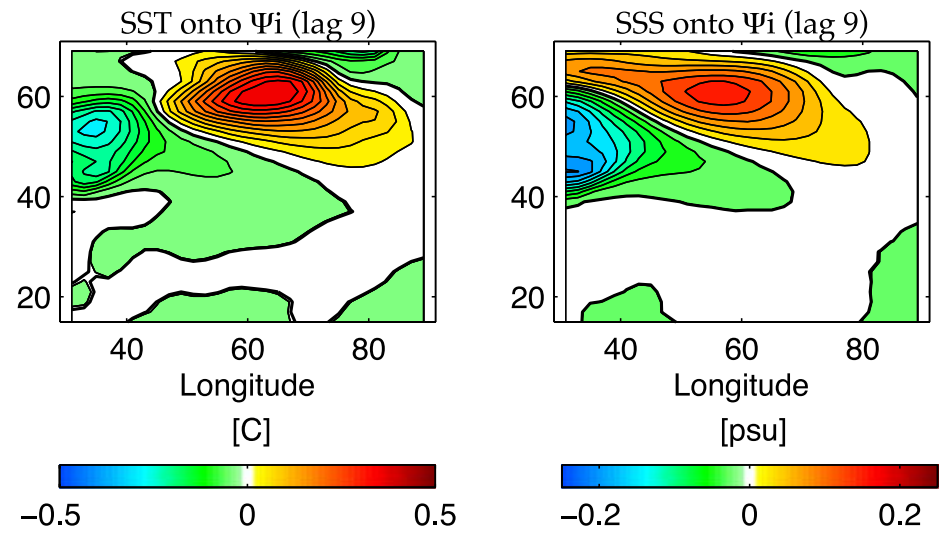

[psu]

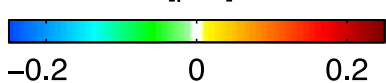

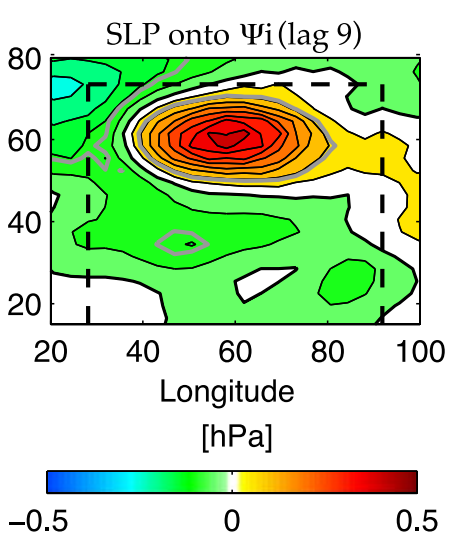

Fig. 7 Linear regression of various quantities onto the MOC index $\Psi_{i}$ in the Northern Hemisphere at different lags during a half oscillation. At negative lags the spatial patterns are of opposite sign and the magnitudes poorly damped in all fields. (Left panels) SST $\left({ }^{\circ} \mathrm{C} \mathrm{Sv}^{-1}\right)$, (middle panels) SSS (psu Sv${ }^{-1}$ ), and (right panels) SLP $\left(\mathrm{hPa} \mathrm{Sv}{ }^{-1}\right)$. The dashed box in the SLP panels represents the ocean basin and the grey contour the 95\% confidence level from a Student's $t$ test

Thus, we see in our model the capacity of the ocean to force and drive the atmosphere at interdecadal time scales, imprinting itself on a particular teleconnection pattern. It is notable that the SST anomalies are, on these timescales, being felt in the mid-troposphere, not just in the lower atmosphere and boundary layer. Specifically, the atmospheric response to this forcing is equivalent barotropic, with a sensitivity of about $20 \mathrm{~m} \mathrm{~K}^{-1}$ at $500 \mathrm{mb}$ (not shown-but see Sect. 5.1 for a discussion on the atmospheric response to time-varying SSTs). This equivalent barotropic stationary wave mode has the strongest response in its northern centre of action, with warm SST forcing a high anomaly at the northern end of the basin (see bottom panels in Fig. 7). This seems consistent with Czaja and Marshall (2001) observational findings of a stronger response in the Greenland-Iceland low, the northern centre 
Fig. 8 (Upper panels) First two canonical correlation patterns (CCP) between SST and SLP in the Northern Hemisphere. SST is shaded with white contours at interval of $0.05^{\circ} \mathrm{C}$, zero contour omitted. SLP is contoured, dashed when negative; contour interval is $0.2 \mathrm{hPa}$, zeros have a heavy solid line. (Lower panels) Same as for the upper panels but for the $450 \mathrm{mb}$ geopotential height (Z450) correlated with SST. Contour interval is $2 \mathrm{~m}$ for Z450. Correlation coefficients (CC) are at the top of each panel
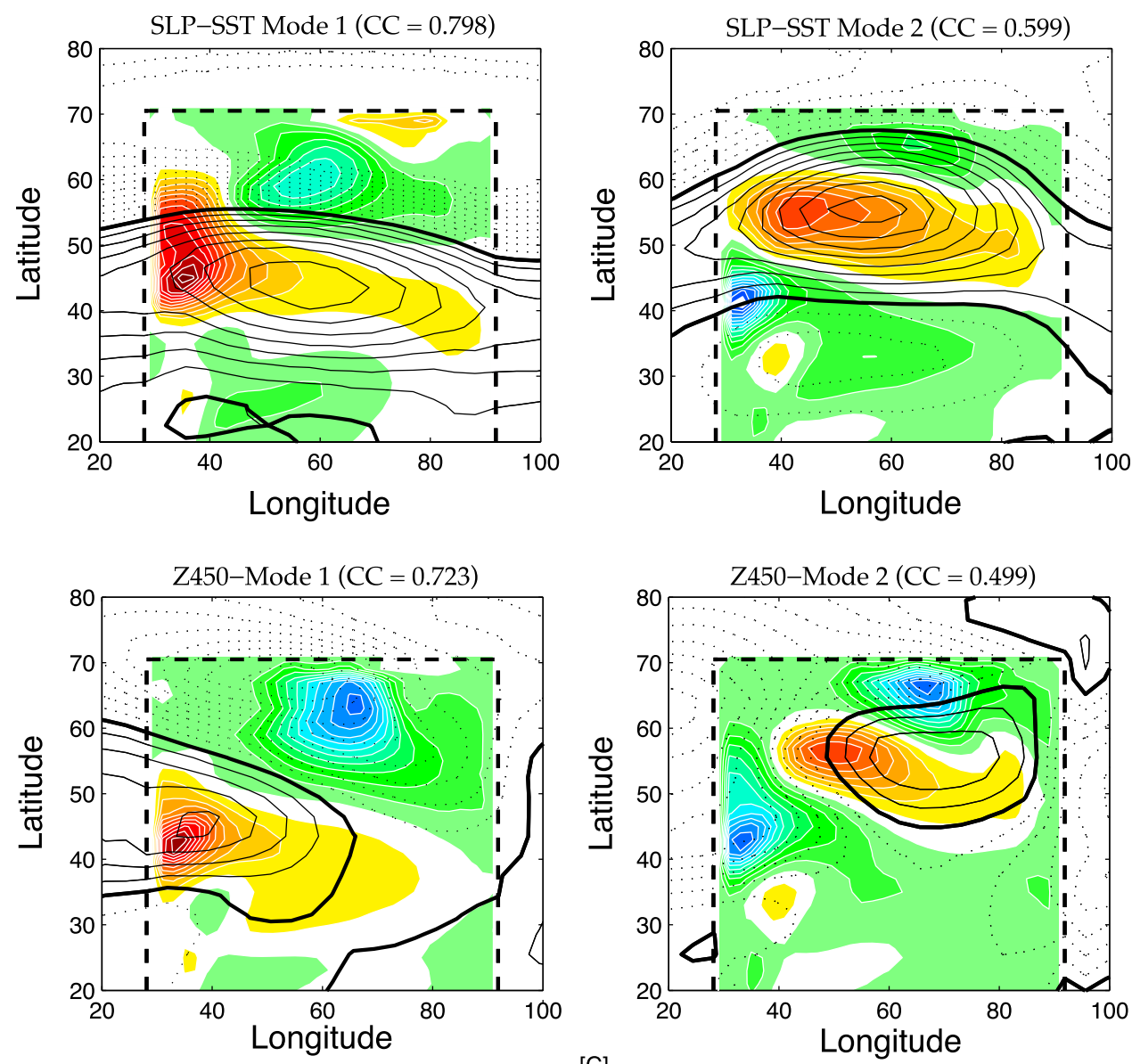

[C]

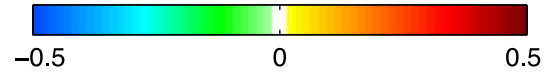

of action of a dipolar SLP pattern modulated by decadal SST anomalies.

A canonical correlation analysis (CCA; von Storch and Zwiers 2001) is performed in order to isolate the low-frequency patterns of maximum spatial correlation (CCP) between SST and the atmosphere aloft. The original data sets were pre-filtered by an EOF analysis to help emphasize true correlations (Preisendorfer and Mobley 1988). Only the first six 5 year low-passed re-normalized EOF patterns were retained for the CCA analyses. The first and second CCP of maximum correlation between SST and SLP resemble their respective EOF-1 and 2 (upper panels in Fig. 8), thus corresponding to a positive and negative phase of the MOC oscillation, respectively. There is a significant spatial coherence of warm (cold) SST anomalies underlying high (low) SLP. Also, the atmospheric equivalent barotropic structure is evident in a similar analysis for the $450 \mathrm{mb}$ geopotential height (Z450) against SST (Fig. 8, lower panels).

To demonstrate that SST anomalies do influence the atmosphere on decadal timescales in the model is important for two reasons. First, and obviously, SST anomalies must be felt by the atmosphere if the ocean is to have an influence on climate. Second, if, on long time periods (e.g., decadal), a primary response to a warm ocean is for the atmosphere itself to warm, then the effect of an interactive atmosphere would be to reduce the effective thermal damping felt by the ocean. On shorter timescales (e.g., annual and shorter) the effect is reversed, in that if the atmospheric variability is the primary driver then the effective thermal damping of the atmosphere is reduced, as described by Barsugli and Battisti (1998) and Czaja et al. (2003). In either case, coupling leads to a greater thermal variance in the system, and potentially an enhancement of the low frequency variability of the ocean on decadal times, and of atmospheric variability on seasonal timescales. Thermal equilibration will turn out to be crucial for the reduced damping of the oscillation in a coupled framework. A good degree of thermal correlation in the CTL coupled experiment is revealed by a canonical correlation analysis between the SST and the free atmospheric temperature at $450 \mathrm{mb}$, after band-pass filtering the data to allow only timescales between 5 and 100 years (Fig. 9). This would 


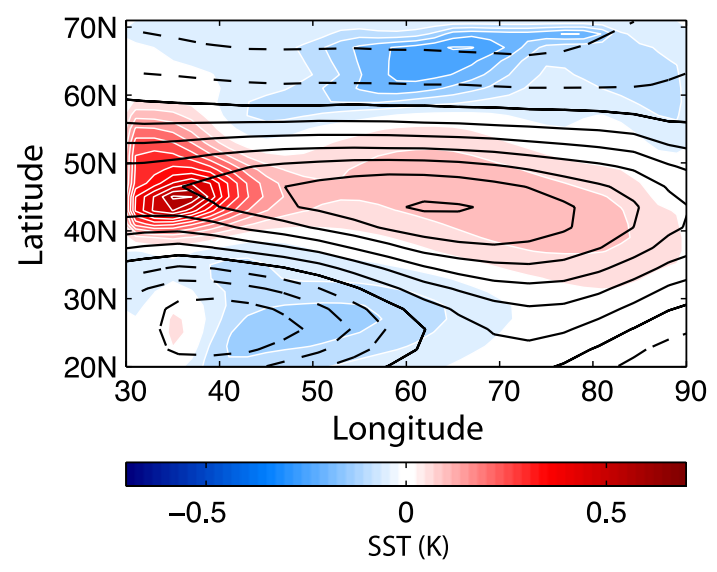

Fig. 9 Patterns of maximum correlation between atmospheric temperature at $450 \mathrm{mb}$ (contoured every $0.1^{\circ} \mathrm{K}$, dashed when negative) and SST (shaded) over the northern half of the ocean basin from a canonical correlation analysis of the CTL coupled experiment. The correlation coefficient between the temperature fields is 0.56

suggest that damping on the ocean on these timescales may indeed be reduced.

\section{The effect of ocean vertical diffusivity and geometry on the variability of the system}

In this section we pose the question: how important is the oceanic circulation in setting up the oscillation? We will explore this issue in two separate modifications of the CTL configuration: by changing the vertical diffusivity $\kappa_{v}$ in the ocean and therefore generating a different mean state, and by doubling the width of the ocean basin. Although we will only focus on a subset of our results, we integrated six 1,000 year long coupled experiments with different $\kappa_{v}$, and one 1,000 year experiment with a twice-as-large basin. All experiments were spun-up to equilibrium prior to the following analysis (see Table 1).

Saravanan and McWilliams (1998) proposed an advective mechanism for ocean-atmosphere interaction in which a preferred time scale exists in the coupled system without requiring the presence of oscillatory modes in the atmosphere or the ocean. In their analytical model, which is restricted to the upper ocean and does not depend on the existence of a meridional overturning cell, oceanic horizontal advection and the length scale of air-sea spatial

Table 1 Different integrations of the coupled model

\begin{tabular}{lllllllll}
\hline Name & Kv005 & Kv01 & Kv025 & CTL & Kv1 & Kv2 & Kv5 & X2 \\
\hline$\kappa_{v}$ & 0.05 & 0.1 & 0.25 & 0.5 & 1.0 & 2.0 & 5.0 & $0.5(2 \times$ wide $)$ \\
\hline
\end{tabular}

All experiments differ only on the value of their constant vertical diffusivity $\left(\kappa_{v}\right.$, given in $\left.10^{-4} \mathrm{~m}^{2} \mathrm{~s}^{-1}\right)$ or ocean width. All experiments were run for 1,000 years following a 2,500 years spin up interactions set the period of the oscillation. The low diffusivity experiment $\mathrm{Kv} 005$ ( $\kappa_{v}$ is $1 / 10$ th of the CTL value) approaches the limit of no meridional cell and air-sea feedbacks are restricted to upper ocean dynamics. In the case of weak meridional circulation the model essentially produces red noise, suggesting merely an integration of atmospheric white noise forcing (grey line in Fig. 10). The oceanic meridional circulation is too weak and localized (not shown) to support the oscillation and the SST anomalies are presumably damped locally before been able to set up a preferred time scale and force an atmospheric response. As a result, the SLP spectra for the Kv005 experiment do not show any significant forced/coupled peak at any preferred interdecadal period. Although advection of anomalies by the mean flow is a crucial component of the variability in our coupled integrations, we can conclude that Saravanan and McWilliams (1998) advective mechanism is not a viable source of interannual and longer variability in our model. In the absence of an oceanic oscillatory mode, local thermal damping effects are dominant over advection, resulting in oceanic variability that can be described as red-noise (Hasselmann 1976). We will return later on the nature of the oceanic oscillation with uncoupled integrations in Sect. 5.2.

The opposite picture is given by the Kv5 simulation (10 times the CTL value; red line in Fig. 10). The vigorous and unrealistically strong MOC (up to $40 \mathrm{~Sv}$ in its northern cell) generates a powerful wide peak at a similar period, but with more variance than CTL. Moreover, the coupled oscillation is recovered, with SST-SSS dipolar patterns flipping the equivalent barotropic atmospheric standing wave at the resonant period. Note also for the Kv5 MOC spectrum the sharp decrease in power at longer interdecadal periods. Similar 'blue' behaviour at longer time scales was also present in CTL, but the departure from a red noise process was smaller. If we consider that in Kv005 and Kv01 the reduction of energy level from the red noise at low frequencies is negligible, we might suppose that the ocean circulation effects at those time scales are similar to those described in Czaja and Marshall (2001) for the case of mean advection. When ocean dynamics is taken into account, there might be a response other than at the resonant time scale at which advective processes act to damp the anomalies in the ocean, thus disabling thermal equilibration and effectively reducing power at those periods in the system.

Finally, the Kv01 and wide basin X2 experiments give similar results though for different reasons. Kv01 (blue line) shows an emerging but still not significant mode around the 20 year period. The background oceanic conditions are close to supporting the oscillation at the advective decadal time scale but no significant atmospheric forcing is possible yet and the mode is heavily damped. As 
Fig. 10 Power spectra of the MOC time series for all the experiments with different vertical diffusivities $(\mathrm{Kv})$ and the wide ocean (X2). See text for details

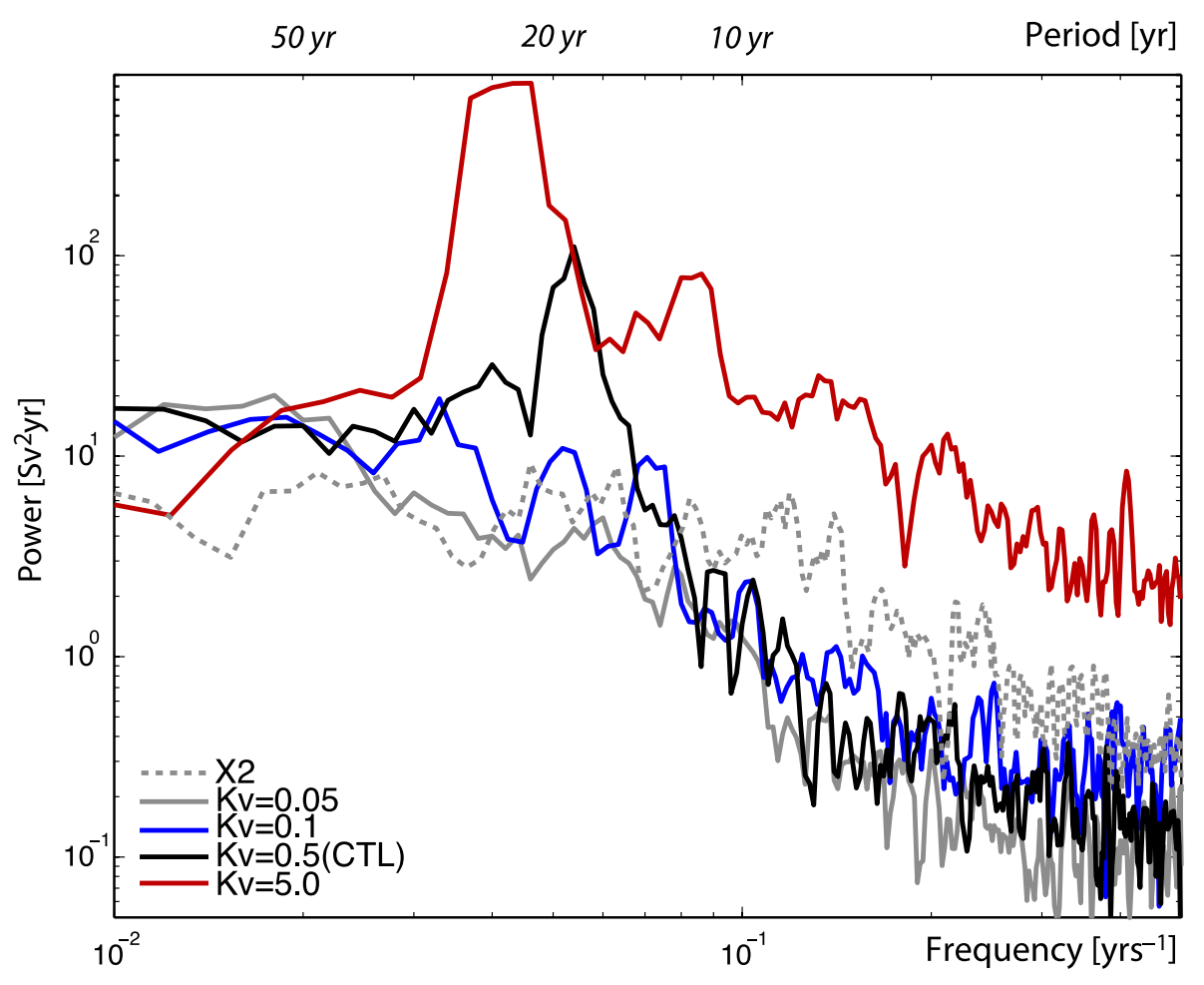

for X2 (dotted grey line), the MOC oscillations are weak and the advection scale from the western boundary to the region of convection is too large to support the buoyancy anomalies that are damped by other mechanisms. Thus, it seems reasonable to suppose that different model formulations and parameterisations - of which vertical diffusivity is just an example-will lead to significant alterations in the period and strength of the oceanic variability, and in turn in its interaction with the overlying atmosphere.

\section{Uncoupled experiments}

Although an overall picture of the natural variability acting in this model emerged from the coupled experiments, a number of questions remain unanswered, as follows. (1) Can the atmosphere or the ocean alone support decadal and longer intrinsic variability and therefore explain the sea level pressure (SLP) spectra shown in the coupled simulations, or is coupling intrinsically necessary? (2) What is the role of that coupling? (3) If, as we posit, the primary source for the oscillation is oceanic, what is its origin? (4) What is the role of the ocean mean state in setting its variability? To address these questions we undertake a number of uncoupled integrations of both the oceanic and atmospheric model component.

We first describe some integrations with a full atmospheric model, coupled to a passive mixed-layer ocean characterized by uniform fixed depth, constant heat capacity, a temperature that varies only in the horizontal and fixed salinity. The mixed-layer model has been integrated in two configurations. In the first, and simplest, the mixed-layer ocean is regarded as a passive swamp, and there are no restoring or flux adjustments at all (experiment denoted ML). In this case we expect to generate a different atmospheric response from the control, coupled, integration because the lack of meridional oceanic fluxes will lead to a larger meridional temperature gradient and a generally more energetic atmosphere. The second configuration (denoted QF) is designed to avoid this by the use of flux adjustments, so-called Qflux, representing the meridional heat flux of a dynamical ocean. In this way we reproduce the same climate as in a coupled experiment, but all oceanic variability is suppressed, thus eliminating any possible oceanic sources of atmospheric variability. The slab ocean model can also be used to provide an SST and sea-ice bottom boundary condition for the atmospheric model, when fixed or timevarying SST and sea-ice concentrations from the coupled experiment are used (experiment AFIX and AVAR, respectively; see Table 2). Several ocean-only experiments were also carried out, as detailed in Table 2. We employed pure flux boundary conditions, in which only the fluxes computed from the coupled run are used to force the ocean without any restoring. To prevent climate drifts in the simulations, sea-ice (and only sea ice) is 
Table 2 Characteristics of the uncoupled integrations

\begin{tabular}{lll}
\hline Name & Run (years) & Surface boundary description \\
\hline Atmos-only experiments & & \\
QF & 500 & Mixed layer ocean with Q-flux \\
ML & 500 & Mixed layer ocean with no flux adjustment \\
AFIX & 500 & No mixed layer ocean; climatological SST from coupled run \\
AVAR & 500 & No mixed layer ocean; time varying SST from coupled run \\
E1 & 150 & As AFIX, but EOF1 of SST is added as an anomaly \\
Ocean-only experiments & & Climatological Coupled Fluxes ( $\mathrm{F}^{c}$ ) of heat, \\
CLIMA & 500 & freshwater and momentum \\
FULL & 500 & Time-varying $\mathrm{F}^{c}$ \\
HEAT & 500 & Time-varying $\mathrm{F}^{c}$ of heat plus climatological \\
& & $\mathrm{F}^{c}$ of momentum and freshwater \\
RAND & 500 & As HEAT but randomly selected $\mathrm{F}^{c}$ of heat \\
RANDDAY & 500 & As RAND but randomly selected daily anomalous $\mathrm{F}^{c}$ of heat \\
\hline
\end{tabular}

restored towards a climatological value with a time scale of 50 days. As we will see, the oceanic behaviour and response to the coupled fluxes varies considerably as $\kappa_{v}$ is modified.

\subsection{Oceanic influence on the atmosphere}

The uncoupled atmosphere-only simulations with a mixed layer ocean or fixed SST gave qualitatively similar results, and none of the atmospheric modes show a distinctive and significant peak at any frequency. The variability has a more-or-less white spectrum behaviour from timescales of years to decades. This general result is common for all the experiments (QF, ML and AFIX) with very similar spatial patterns (and therefore intrinsic modes of variability of our atmosphere) and variance accounted for. Thus, in the absence of active coupling with a dynamic ocean, our atmosphere does not support enhanced variability on decadal and longer timescales. The power found in the coupled case must involve the ocean in some way, either through an oceanic interdecadal mode imprinting itself on the atmosphere or conceivably some form of coupled interaction.

We investigate further the role of SST anomalies and their ability to drive the atmosphere at decadal and longer time scales by looking at the spectra of the oceanic and atmospheric 5 year low-passed annual-mean surface temperatures in the Northern Hemisphere. From Fig. 11 it is evident that the atmosphere shows enhanced variance at the low frequency end of the spectrum when coupled, consistent with the argument of a weaker damping of thermal anomalies in a coupled framework (e.g., Barsugli and Battisti 1998) The atmospheric surface temperature (AST) closely follows the SST spectrum at decadal and longer periods, with a $\sim 20$ year peak consistent with its oceanic

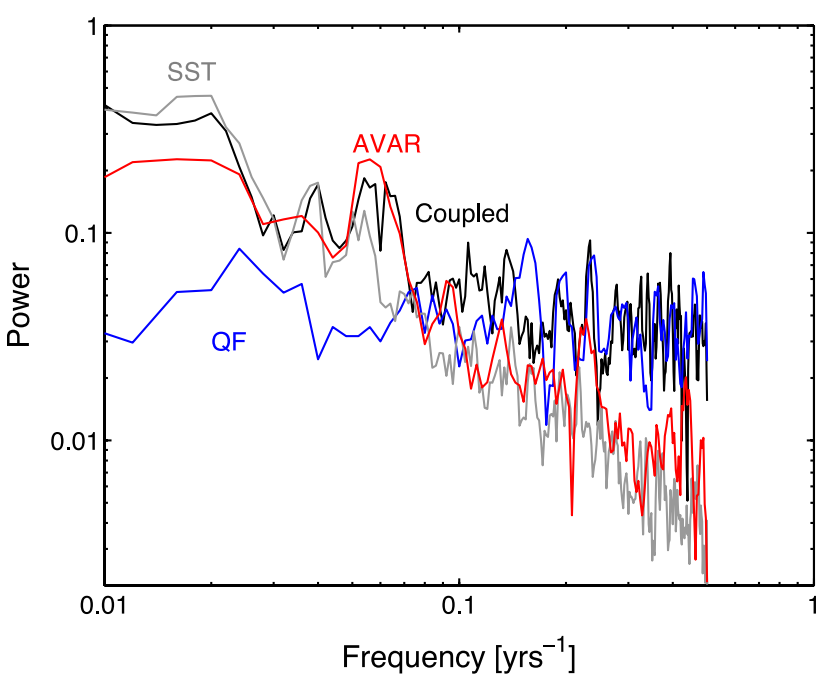

Fig. 11 Power spectra $\left({ }^{\circ} \mathrm{K}^{2}\right.$ year) of atmospheric surface temperature in the Coupled (black), Q-flux (blue) and AVAR experiment (red) averaged over the entire Northern Hemisphere. In the latter experiment, the imposed time-varying SSTs are responsible for the atmospheric enhanced power at interdecadal periods (see text). The SST from the coupled run is plotted in grey

counterpart superimposed on a red noise structure. In contrast, the uncoupled atmospheric spectrum (experiment $\mathrm{QF})$ is virtually white at all frequencies.

SST anomalies produce an atmospheric response of the same sign, modifying the atmospheric meridional temperature gradient and therefore its baroclinicity, giving rise to compensatory effect in the atmospheric meridional heat transfer. This variability occurs on the decadal timescale and is not present in any uncoupled simulation, showing that it is not a manifestation of the atmosphere driving the oceanic variability at shorter timescales. To further demonstrate the ability of the atmosphere to respond to the 
ocean on long timescales, we perform an integration of the atmospheric model forced by time varying, annually averaged, SSTs taken from the coupled run (experiment AVAR). The atmosphere is found to respond to the oceanic oscillation and the enhanced power at decadal and longer periods is recovered (Fig. 11, red line). Also, the mid-latitude SSTs project more strongly on the second mode of
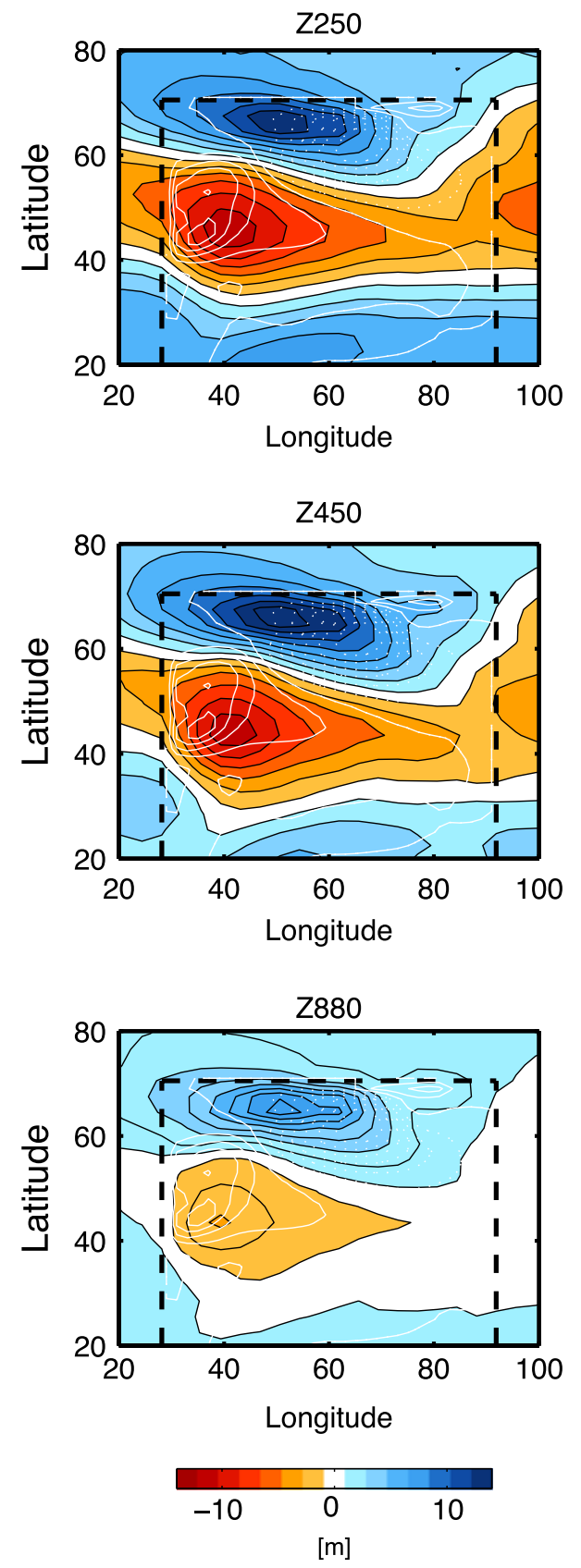

Fig. 12 Atmospheric response patterns to the SST principal mode of variability from the coupled CTL run at 250, 450 and $880 \mathrm{mb}$. Contour interval is $2 \mathrm{~m}$. The SST EOF1 pattern forcing this response is contoured in white, dashed when negative, with $0.1^{\circ} \mathrm{C}$ contour interval. The dotted black box denotes the ocean basin variability and the second mode shows a statistically significant peak at the oscillation period as in the coupled solutions (not shown).

The pattern of the atmospheric response is further investigated when the first EOF of the SST from the coupled run is added as a steady anomaly to the SST climatology (experiment E1). The difference between the time mean E1 and AFIX solution will give an atmospheric residual that is the response to the primary mode of variability in SST. Results for the geopotential height at 880 , 450 and $250 \mathrm{mb}$ are given in Fig. 12. As in the coupled results, the response is a dipole, equivalent barotropic in structure, with highs (lows) over warm (cold) anomalies. Our results of the atmospheric response to SST anomalies in the mid-latitudes seem consistent with recent modelling studies (Ferreira and Frankignoul 2005; Cassou et al. 2007; Deser et al. 2007).

\subsection{Atmospheric fluxes exciting the ocean}

In this section, we turn our attention to ocean-only integrations with different imposed surface fluxes (Table 2) in order to isolate the origin of the oscillatory mode. We performed experiments for three different values of the oceanic vertical diffusivity, namely the lowest, Control and highest case.

Figure 13 shows the results for the CTL case $\left(\kappa_{v}=\right.$ $\left.0.5 \times 10^{-4} \mathrm{~m}^{2} \mathrm{~s}^{-1}\right)$. In experiment CLIMA, where all fluxes are kept constant at their climatological value, the MOC time series, after a few years of adjustment, settles around a value of $\sim 10 \mathrm{~Sv}$ and no oscillations are found (top panel, dotted black line). Evidently, for these parameter settings, the model ocean does not support any intrinsic instability and some external forcing is needed for the oscillation to develop.

If the ocean model, at least in this parameter setting, is not intrinsically unstable, what are the fluxes that excite its variability? If the annual mean fluxes from the coupled experiment are saved and then used to force the ocean then the oscillation is recovered (experiment FULL, blue line in top panel of Fig. 13), reproducing almost exactly the oscillation in the MOC of the coupled model with a high correlation between the two $(0.82)$ and very similar variance (1.46 Sv for Coupled and 1.45 Sv for FULL). With hindsight, this result is perhaps not surprising: the time series of the annual mean coupled surface fluxes carry information of the coupling and increased variance at the period of the variability. Also, we find that forcing the ocean with time-varying surface heat fluxes only is sufficient for generating the oscillation (HEAT, blue line in bottom panel of Fig. 13). Both the correlation between the Coupled and HEAT experiment and the variance are very similar to the full flux forcing FULL, suggesting that 
Fig. 13 Annual mean MOC time series for the last 500 years of the Coupled CTL experiment (thin black line in both panels) and the uncoupled, ocean-only experiments with different forcing fluxes. See Table 1 for more information on the forcing fields
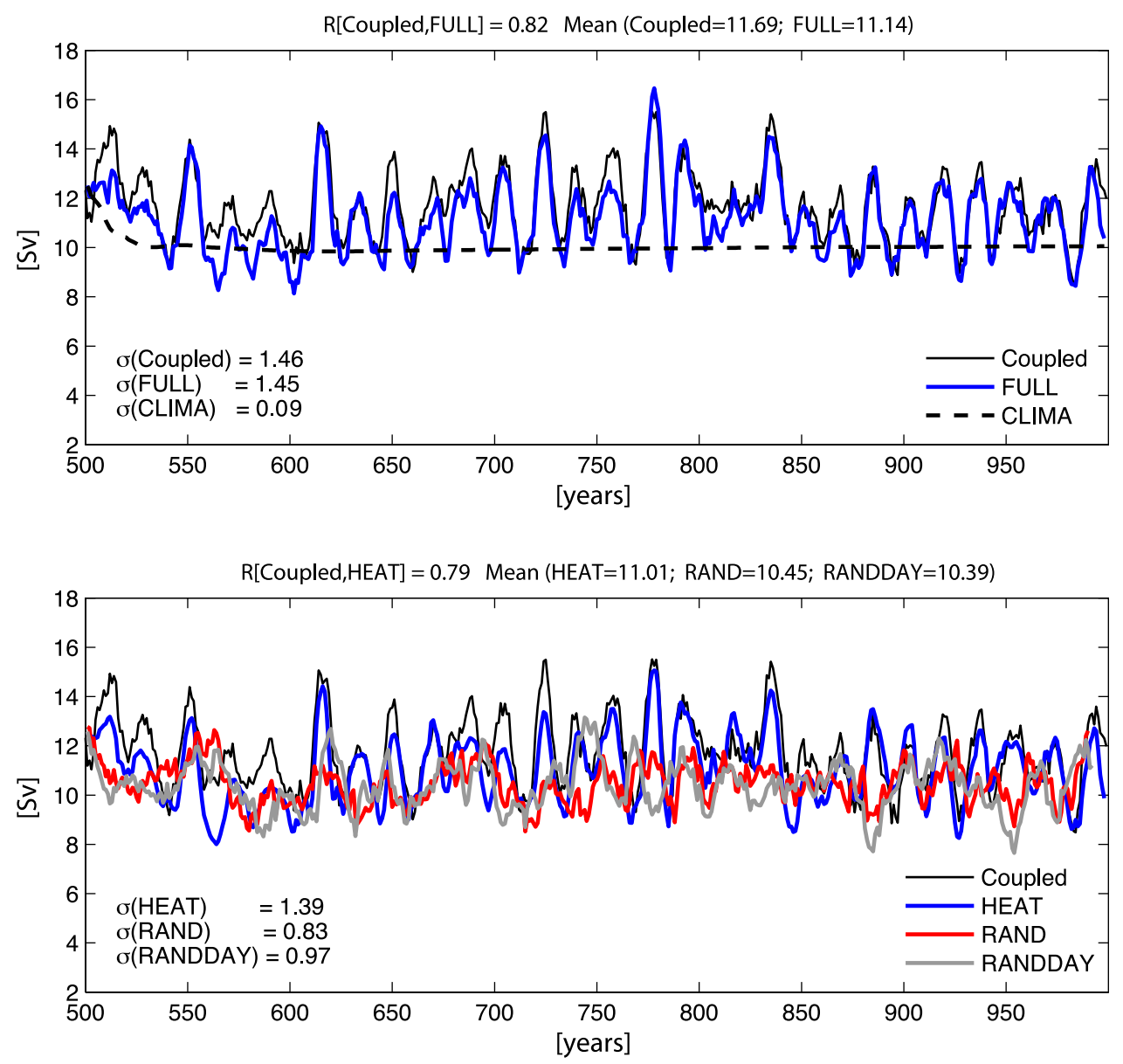

momentum and freshwater fluxes play a secondary role in destabilising the ocean circulation, as also found by Griffes and Tziperman (1995) and Delworth and Greatbatch (2000). Hence, we will focus on the effect of the heat fluxes for the remaining of the paper.

The effect of stochastic forcing is analyzed through randomly selected heat fluxes from the coupled run, either annual or daily. In this way we ensure that the fluxes do not have any correlation from the coupled run. The results do show some interdecadal variability in the ocean, although not as much as in the fully coupled experiments (bottom panel of Fig. 13; red line for annual (RAND) and a grey line for daily (RANDAY) anomalies). Substantial variability is generated in the MOC for these forcings, with increasing variance for the case of daily anomalies ( $0.83 \mathrm{~Sv}$ for RAND and 0.97 for RANDAY), although still significantly smaller than when the full coupled fluxes are used. Thus, when forcing with purely random heat fluxes, the ocean responds generating interdecadal variability, although with rather different properties than when active coupling is taking place. The ability to modify the amplitude of the MOC variability by daily anomalies in RANDAY suggests a role for stochastic high-frequency atmospheric noise. The Fourier spectra of all experiments summarise the previous findings (Fig. 14, top panel). FULL and HEAT are able to reproduce the significant coupled spectral peak, while experiments RAND and RANDDAY have power at multidecadal periods but this is not significant. We conclude that, although interdecadal variability can be excited by random atmospheric heat fluxes, the large reduction in amplitude suggests that such forcing should not be regarded as the primary cause for the existence of the oscillation in the coupled experiments.

From Figs. 13 and 14 we can infer that air-sea coupling undoubtedly does play a major role in modulating the amplitude of the oscillation and, to a lesser extent, its period. This is confirmed by the autocorrelation function (ACF) of $\Psi_{i}$ in the different coupled and uncoupled settings (Fig. 14, bottom panel). The period of the oscillation is essentially unchanged but the e-folding time scale increases in the uncoupled simulations when the ocean is forced with heat fluxes only. The importance of the previous result is only in the ability to reproduce the coupled 

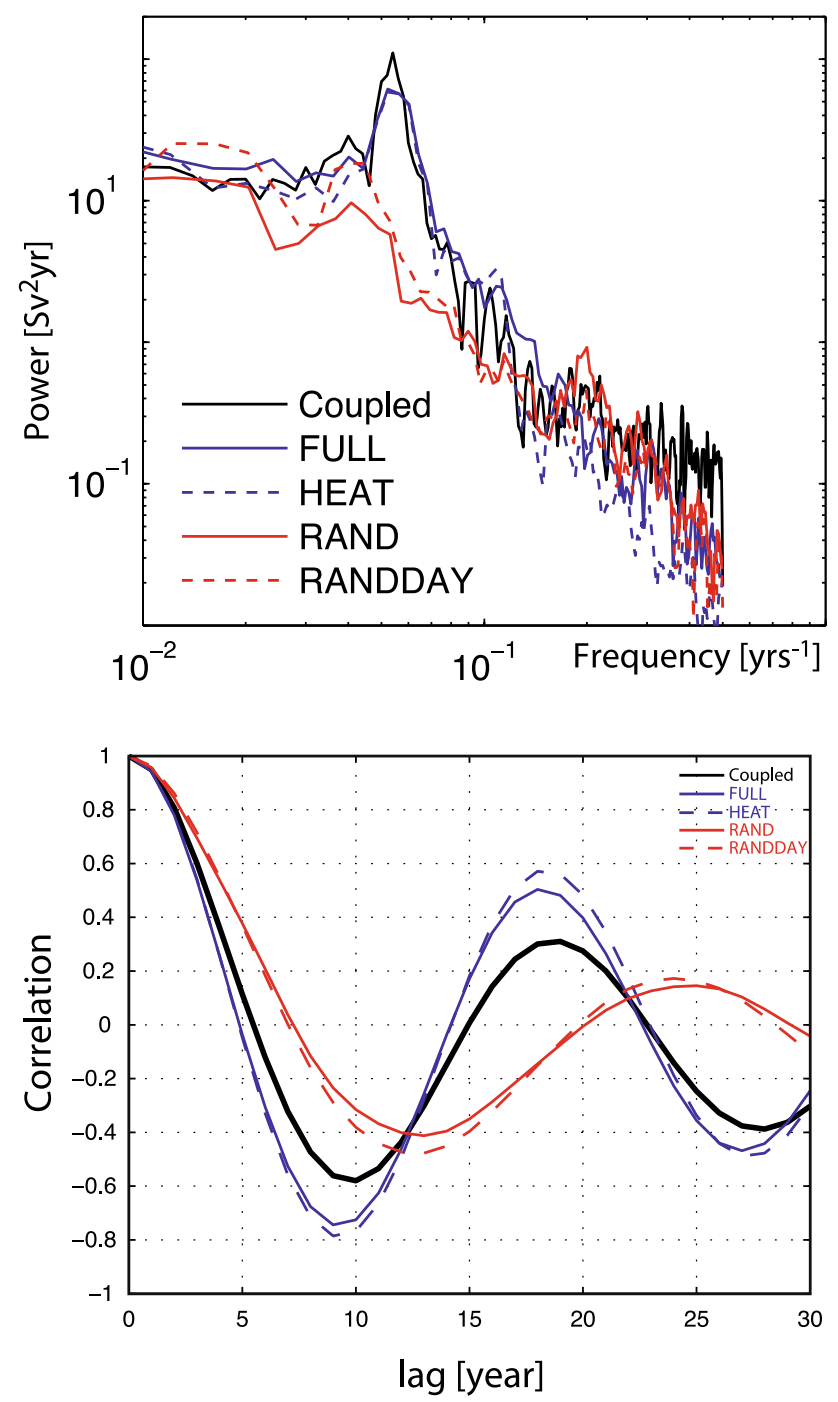

Fig. 14 (Top) Spectra of the annual mean time series shown in Fig. 13. Experiments were the coupled fluxes are used to force the ocean (FULL or HEAT) are able to reproduce the interdecadal mode of variability. When stochastic forcing is used (RAND or RANDDAY) some interdecadal variability is excited but with much less power. (Bottom) Autocorrelation functions of the MOC time series for the CTL coupled and uncoupled cases. Similar timescales are found for the Coupled, FULL and HEAT simulations with minima al lags $\sim \pm 10$ years, hence a period of about 20 years. For the randomly forced experiments RAND and RANDDAY the oscillation is moved towards longer periods ( $\sim 25$ years $)$ and is more damped

oscillation with coupled surface fluxes and in finding the optimal forcing agent, in this case fluxes of heat, that generate an even greater oscillation in the absence of disturbing air-sea interactions. A different picture emerges when the system is stochastically forced, with no a priori information of the oscillation in the fluxes. ACFs have a larger period with minima at lags \pm 13 years and are in a higher damped regime (red lines in Fig. 14), implying that coupling sustains the oscillation against dissipation.

\subsection{The role of the ocean mean state}

The above analyses were all performed using the CTL value of $\kappa_{v}$. We previously discussed the importance of the background oceanic mean state in supporting the interdecadal mode of variability and in forcing an atmospheric response. For this reason we now explore the ocean-only behaviour of the two extreme cases in the strength of the circulation: $\mathrm{Kv} 005\left(\kappa_{v}=0.05 \times 10^{-4} \mathrm{~m}^{2} \mathrm{~s}^{-1} ; 1 / 10\right.$ th of the CTL value) and $\mathrm{Kv} 5\left(\kappa_{v}=5.0 \times 10^{-4} \mathrm{~m}^{2} \mathrm{~s}^{-1} ; 10\right.$ times the CTL value).

The circulation is both weak and weakly oscillatory for the lowest value of diffusivity, with the ocean behaving mainly as a simple integrator of atmospheric noise resulting in a red noise oceanic spectrum (not shown). This suggests that the fluxes have no direct role in determining the oscillation, but rather are acting simply as a source of noise, producing oscillations in a similar fashion to the box model of Griffes and Tziperman (1995). On the other hand, Kv5, has an unrealistically strong constant vertical diffusivity, and its coupled and uncoupled circulations are illustrated by Fig. 15. In this case, when forced with climatological time mean fluxes, the model does produce a regular oscillation of about 25 years, although the amplitude of the MOC is modest compared to the coupled solution (a standard deviation of $1.43 \mathrm{~Sv}$ is found in CLIMA while in Coupled was more than doubled, $3.97 \mathrm{~Sv}$ ) and the strength of the mean overturning circulation is, interestingly, considerably weaker when the model is uncoupled. This leads to the conclusion that air-sea coupling is indeed important in setting the magnitude of both the time mean MOC and its anomalies, but that the underlying oceanic oscillation is self-sustained in the Kv5 mean state and that coupling merely amplifies its manifestation. The spectra of the Coupled and CLIMA annual mean MOC time series indeed present similar variance at similar interdecadal periods with minor modulations in the period and power when the system is coupled.

Very different behaviours were observed in the variability of the coupled system when the ocean background diffusivity $\kappa_{v}$ was modified, and hence the response of the uncoupled oceans to the same realization of their respective coupled fluxes when three oceanic states were taken into account. Thus, we will try to classify the different oceanic responses to coupling by the oceanic mean state. When the ocean is integrated with parameters that give most similarity to present-day conditions in the Atlantic ocean, namely with a medium range value of $\kappa_{v}=0.5 \times$ $10^{-4} \mathrm{~m}^{2} \mathrm{~s}^{-1}$, it does respond, albeit weakly, to stochastic excitation from imposed, variable, atmospheric fluxes, suggesting that it has some of the properties of a damped oscillator that can be stochastically excited, as in Griffes and Tziperman (1995). However, the coupling to an 

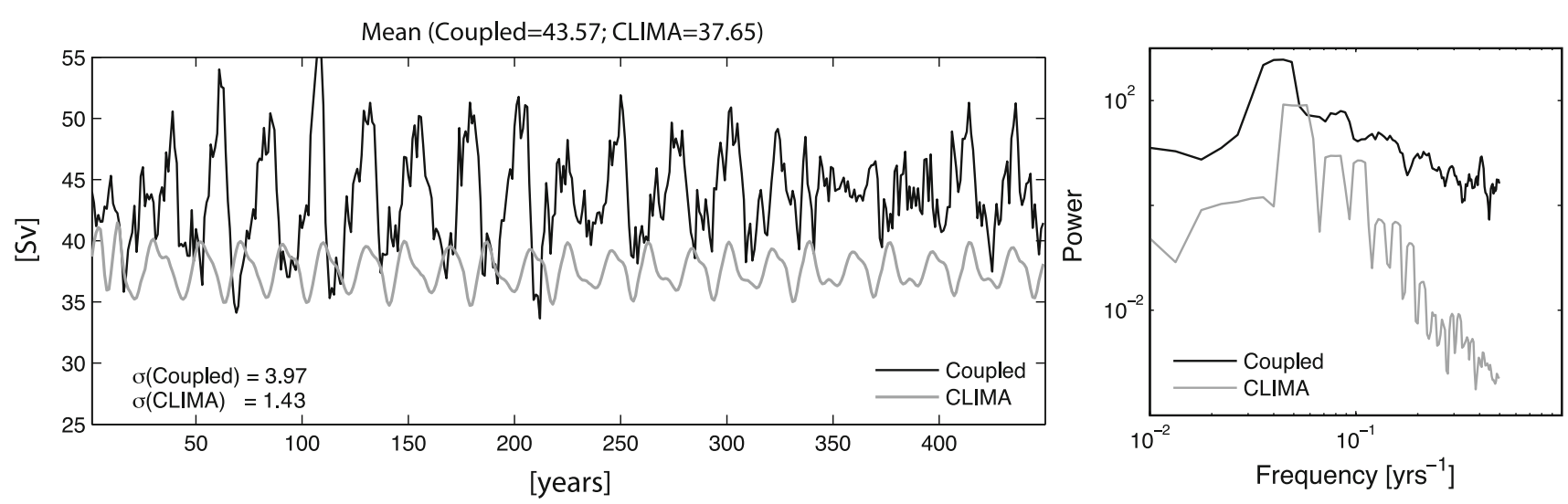

Fig. 15 Annual mean MOC time series for the Kv5 experiment $\left(\kappa_{v}=5.0 \times 10^{-4} \mathrm{~m}^{2} \mathrm{~s}^{-1}\right)$. Only the result of the fixed climatological fluxes (CLIMA) are shown. The corresponding spectra are in the right panel

atmosphere has the strongest effect in sustaining and amplifying the oscillation. This suggests that the ocean is sensitive to the amount of surface damping and, by having an interactive atmosphere, this damping is reduced. For a very low diffusivity and a weak diffusively driven circulation, the coupled model produces rather weak oscillations, and no regular oscillation is excited by the random atmospheric fluxes. There is no preferred time scale in the oceanic variability and it is not capable of generating coherent and sufficiently strong surface anomalies to influence the atmospheric state. In this case the ocean is just an integrator of atmospheric white noise and its spectrum is reddened by the coupling. In contrast, when the oceanic diffusivity is high the overturning circulation is vigorous; now the ocean is intrinsically unstable and oscillations are generated spontaneously without the need for external forcing. However, just as in the medium diffusivity case, coupling to an atmosphere again enhances the amplitude of the oscillations.

To summarize, moving through the $\kappa_{v}$ space from low to high values we are changing the regime of the oscillation and spanning many of the mechanisms of air-sea interactions at decadal and longer timescales that actively involve the ocean, as defined in the Introduction. Other factorssuch as horizontal diffusivity, viscosity and resolutionmay certainly be important in setting the variability of the system, but of these the oceanic diapycnal diffusivity is the parameter that most immediately affects the structure of the MOC (Vallis 2006).

\section{Discussion and conclusions}

Coupled and uncoupled experiments performed with a climate model of intermediate complexity have been presented. The advantage of such a model over a more comprehensive GCM is that a greater parameter regime can be studied and robust processes identified more cleanly. The disadvantage is that the model is less realistic than a full GCM, so that care must be taken in the interpretation of the results and in ascribing physical reality to them. Integrations with the idealized coupled model showed a quasiperiodic oscillation of about 20 years in the ocean with a strong dependence on the background vertical diffusivity and the dimensions of the basin. The MOC oscillations are accompanied by in-phase surface buoyancy anomalies propagating polewards towards the region of convection, where temperature contributions to density anomalies dominate over salinity. As the ocean varies on decadal time scales the atmosphere responds to the anomalous oceanic heat transport in a quasi-equilibrium manner and the variability is imprinted on the atmosphere both at the surface and aloft. The amplitude of the oscillation and to a lesser extent its period was found to depend on the oceanic vertical diffusivity parameter, with increasing power as $\kappa_{v}$ increases. The nature of the variability changes from a red noise behaviour with no dominant peak at low diffusivity to an increasingly powerful preferred mode of variability at high diffusivity. The oceanic oscillation is found to project weakly but significantly on an atmospheric pattern of variability characterized by a meridional dipole, generating power at interdecadal time scales. The atmospheric response to the anomalous SST dipole is equivalent barotropic, with warm highs (cold lows) over warm (cold) ocean anomalies, and the positive and negative phases of the atmospheric dipole are found to covary with the oceanic mode and share the same interdecadal peak.

For sufficiently strong oceanic circulations, the mechanism for the interdecadal oscillation is schematically summarized in Fig. 16. Suppose we are in a positive phase of the MOC, with an anomalous strong circulation and an SST dipole as shown by EOF-1 of Fig. 3. Warm salty anomalies are generated at around $40^{\circ} \mathrm{N}$ and subsequently advected by the mean flow towards higher latitudes. This 


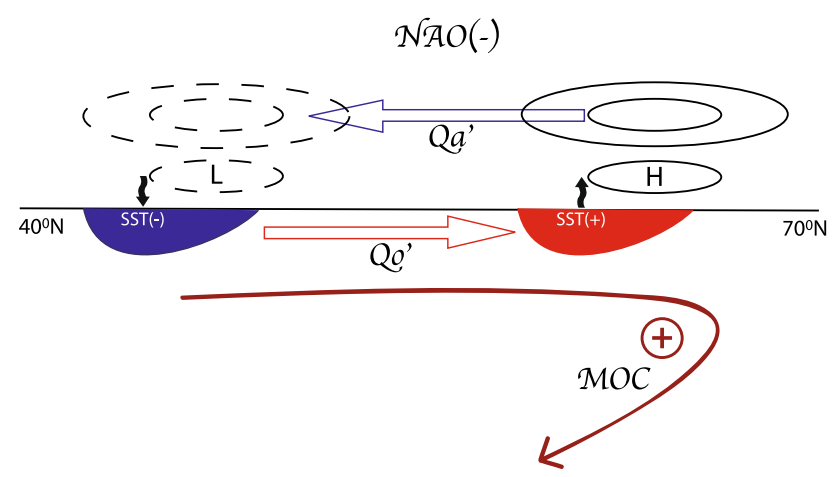

Fig. 16 Schematic of the extratropical ocean-atmosphere interdecadal interactions in the model. The heuristic explanation should be read from the bottom (ocean) to the top (atmosphere). During an anomalously strong MOC, warm salty waters are advected northward by the mean flow after generation through perturbation advection; this leads to an anomaly in the oceanic meridional heat flux $\left(Q_{o}^{\prime}=\bar{v} \theta^{\prime}\right)$ raising SSTs in the North. The anomalous SST dipole forces an equivalent barotropic response in the troposphere, with warm oceanic temperatures SST(+) underlying warm anticyclonic SLP $(\mathrm{H})$, and vice versa. Thus, the atmosphere enters into a negative NAO-like pattern with an associated heat flux anomaly $Q_{a}^{\prime}$ to the south, compensating for $Q_{o}^{\prime}$. Now, both the warm SST anomaly and the surface heat fluxes conspire to revert the cycle

temperature advection implies a northward positive anomaly in the oceanic heat flux $Q_{o}^{\prime}$. The high latitude ocean becomes warm and salty, with the warmth dominating over the salinity and producing a negative density anomaly. The MOC then starts diminishing in intensity resulting in negative SST anomalies and the opposite phase of the cycle can now start. The SST anomalies produce an atmospheric response, which is equivalent barotropic with highs over warm and lows over cold anomalies. Thus, a warm ocean is accompanied by a warm atmosphere, implying a reduced thermal damping of the buoyancy anomalies and supporting the oscillation against dissipation.

When the high-latitude SST anomalies are positive the atmospheric response is akin to the negative phase of the meridional dipole. There is then a reduced atmospheric baroclinicity and a negative anomaly in the meridional atmospheric heat flux $Q_{a}^{\prime}$, so partially compensating for the anomalously strong meridional heat flux in the ocean. That is, there is an anticorrelation between the meridional oceanic and atmospheric heat transports at decadal time scales in the extra-tropics, as first hypothesized by Bjerknes (1964). A time series of the heat transport anomalies in the ocean and atmosphere is illustrated in Fig. 17 for the Northern Hemisphere. It is seen from Fig. 17 that the variations in the strength of the MOC tend to lead the variability in the ocean heat transport. The anomalous heat transports time series are strongly anticorrelated $(-0.71)$ and the atmosphere is compensating, although imperfectly, for variations in the ocean transport.

In our model the timescale of the reversal of the oscillation is determined by advective processes involving the MOC, with horizontal gyre interactions of somewhat lesser importance. The oscillation involves ocean-atmosphere coupling in that an interactive atmosphere is necessary in order to produce a reduced thermal damping-otherwise the oscillations are damped and the system goes to a steady state - but no dynamical interaction between the ocean and atmosphere is needed. Note that it is of little importance whether we identify our primary atmospheric response to the oceanic oscillation as the Atlantic NAO dipole or EAP monopole. What matters here is the ability of the ocean to drive the atmosphere at interdecadal periods, and the subsequent positive feedback (or reduced thermal damping) that the latter can provide. Recent CGCMs studies have
Fig. 17 Variability of the oceanic $\left(Q_{o}^{\prime}\right)$ and atmospheric $\left(Q_{a}^{\prime}\right)$ heat transport and MOC (in $\mathrm{Sv} / 100$ ) anomalies over a 500 year period of the coupled control integration, low-pass filtered to allow only decadal and longer period variability. Transports are averaged from $20^{\circ}$ to $70^{\circ}$ in the Northern Hemisphere and $\mathrm{R}\left(Q_{o}^{\prime}-Q_{a}^{\prime}\right)$ refers to the correlation between the oceanic and atmospheric heat transport anomalies

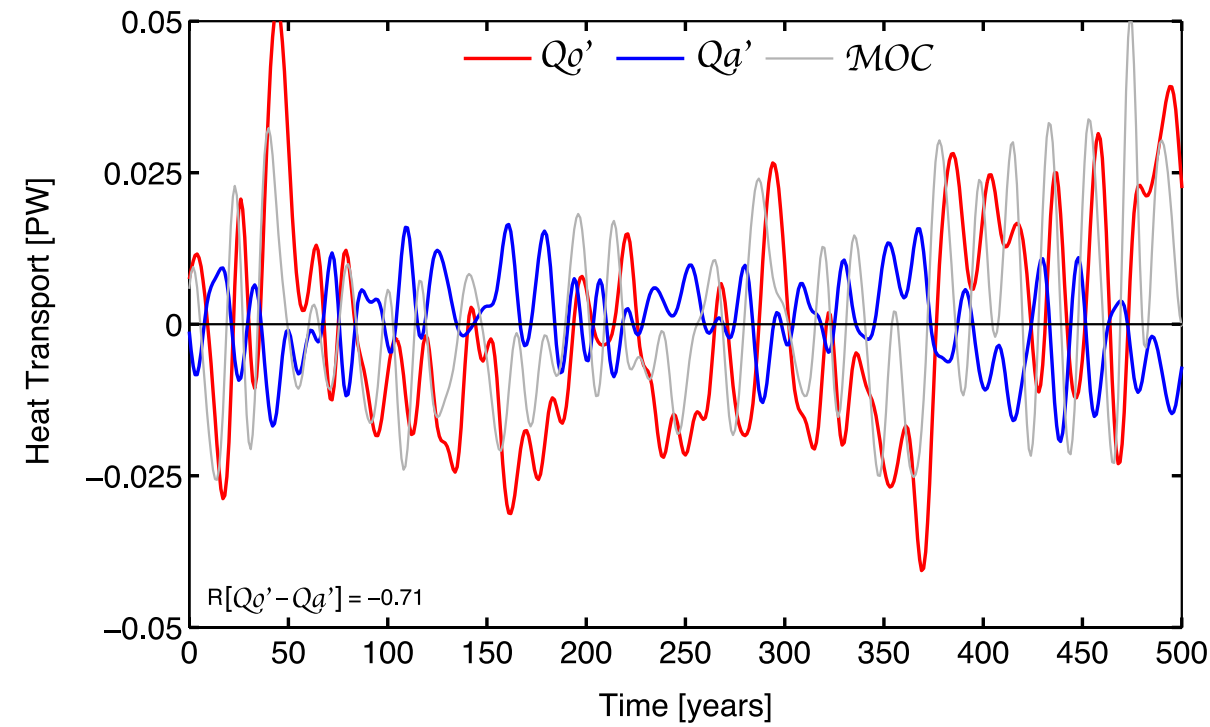


found similar mechanisms of low-frequency air-sea interactions, where the NAO (Bellucci et al. 2008) or the EAP (Msadek and Frankignoul 2008) was the primary pattern responding to oceanic variability through SST forcing.

To shed more light on the role of coupling, several uncoupled integrations were also described with the aim of characterizing the intrinsic variability and origin of the oscillations. Uncoupled atmospheric integrations showed no statistically significant variability at decadal and longer timescales. The atmosphere has an essentially white spectrum, differing significantly from the coupled experiments. This result suggests that the presence of a dynamical ocean is necessary to produce climate variability on time scales of decades and longer in our model, and possibly in the atmosphere. The interdecadal spectral peak and reddening of atmospheric temperatures are reproduced when integrating the atmospheric model with time varying SSTs from the coupled run. The atmospheric response is an equivalent barotropic meridional dipole of significant variability at the oceanic oscillation period, consistent with mid-latitude SST variability imprinting itself on the atmosphere on long timescales. On these timescales the atmosphere essentially reaches a new quasi-equilibrium state.

The oscillation is rooted in the ocean and depends strongly on its mean state. In the case of very low diffusivity, the ocean behaves primarily as an integrator of random forcing. No preferred period of variability is excited and there is little feedback on the atmospheric state. If the ocean model is integrated with a large diffusivity, producing a very vigorous overturning circulation, then instabilities in the MOC express themselves spontaneously at decadal-interdecadal periods, even in the absence of an interactive atmosphere and even if the fluxes forcing the ocean are temporally constant. When medium values of diffusivity are used and if the ocean is forced with a steady atmosphere a steady circulation results. If random, timevarying surface fluxes are added then weak decadal-scale oscillations are produced, but they are still of much smaller magnitude than those obtained in the fully coupled integrations. Finally, coupling with the atmosphere enhances the variance of the oscillatory mode present in the ocean and sustains the oscillation against dissipation through reduced thermal damping. The 'slaved atmosphere' acts as a positive feedback on the oscillation since atmospheric and oceanic surface temperatures are in phase, reducing damping of surface thermal anomalies at the preferred oceanic period and allowing the ocean to oscillate more readily. However, the ocean must be predisposed to oscillating: if, for example, the diffusivity is small and the MOC weak, then self-sustained oscillations are weak or non-existent, even with a fully coupled atmosphere. The fact that the decadal scale oscillations have an oceanic origin, are enhanced by coupling with an atmosphere and by having a stronger mean MOC seem to be robust results that we believe are likely to transcend the simplifications inherent in our model.

Our model can be criticized for its simplified geometry and omission of potentially important features (e.g., ocean topography, orography, continental boundaries). For example, the presence of a seasonal cycle could favour a reduction in strength of the variability by burying SST anomalies in the summer and hence altering the damping of surface buoyancy anomalies. However, it is not clear what would be the role of the reemergence of those anomalies and of a stronger atmospheric stochastic forcing in the winter months. The model bridges the gap between full coupled GCMs, that can be unwieldy and difficult to analyze, and much simpler models that are still less realistic and do not fully incorporate many dynamical processes. Although the coupled model used in this study has shown a fairly realistic Atlantic-like climate, the idealized framework clearly makes any direct comparison with observations very difficult. Furthermore, observations are not available on centennial timescales. However, we can compare some of our results to more comprehensive stateof-the-art climate models, and to this end we have analyzed the output of the preindustrial run of the GFDL model CM2.1 (Delworth et al. 2006). Annual mean data for the 2,000 year long run were used to compute the MOC time series, whose anomalies are plotted in Fig. 18 together with its spectrum. The variability in CM2.1 is very similar to that of the idealized coupled model at annual to decadal time periods. The dominant 20-year peak is presentalthough with a decrease in power-and only at centennial timescales do the two models differ significantly, possibly because of the different length in the simulations. To the extent that this indicates similar dynamics, it suggests that the oscillations in CM2.1 arise through coupling with the atmosphere, rather than from a stochastic forced regime, although we do not know what the response would be if the oceanic component was forced with random fluxes. A detailed analysis of this model is underway and is beyond the scope of this paper.

The variability in the MOC was also extensively studied within an earlier version of the GFDL model (CM2.0; Delworth et al. 1993), in which the authors found the lowfrequency stochastic atmospheric forcing to be the driver of their oceanic oscillation. Compared to CM2.1, the MOC in CM2.0 was weaker and had a longer period (about 50 years; not significant at the $95 \%$ confidence level) and possessed a rather different MOC variability than its successor. The oscillation in the Control case described in this paper bears similarities with other CGCMs where active air-sea coupling is suggested (for example, Timmermann et al. 1998; Dai et al. 2005; Danabasoglu 2008), in which 
Fig. 18 Top panel: Time series of the annual mean MOC anomalies for the GFDL CM2.1 control integration, defined as the maximum overturning streamfunction between $20^{\circ}$ and $70^{\circ}$ N. Bottom panel: Power spectra of the GFDL MOC time series (blue line) and those from two integrations of the idealized model presented in this study: the CTL Coupled (black line) and RANDDAY (grey line) experiment [yr]

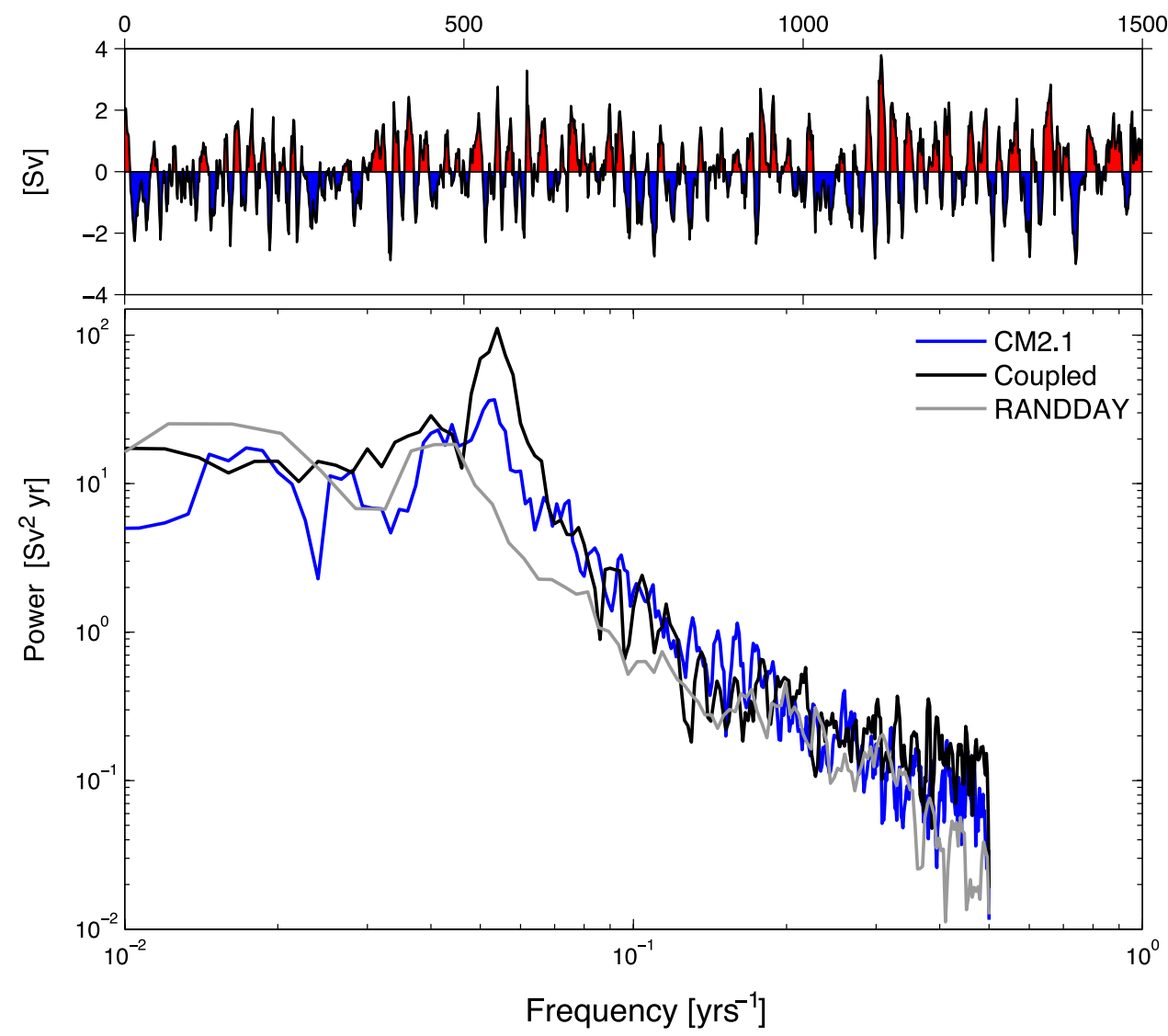

strong regular anomalies in the MOC time series form part of a coupled oscillation with similar interdecadal periods. Many atmospheric GCMs seem to show only a weak response to SST anomalies, and this is not inconsistent with our results: the ocean effectively drives the atmosphere only on long timescales, where a weak signal can emerge from the atmospheric noise. Indeed, on these timescales the atmosphere must respond to changes in the underlying ocean, because on these timescales the atmosphere reaches a new quasi-equilibrium state, and it is not surprising that oceanic changes reach into the mid-troposphere and affect the atmospheric heat transport. Recent results with CGCMs (Bellucci et al. 2008; Msadek and Frankignoul 2008) seem consistent with this interpretation.

In light of these results, we believe many discrepancies on the multi-decadal variability and mechanisms of air-sea coupling across CGCMs could be explained by their different oceanic mean state and formalism. The question remaining is whether the regular quasi-decadal oscillations found in a number of many modelled MOCs do exist in reality. Some recent isopycnal and high-resolution coupled models seem not to show significant interdecadal variability in the ocean, suggesting that a different representation of some physical oceanic processes may have changed radically the variability in the system. A coupled model intercomparison study, spanning a hierarchy of oceanic models with different physics, resolution and complexity, would seem to be needed to unambiguously determine the mechanisms of MOC variability, predictability and its role on ocean-atmosphere interactions at the decadal period and longer.

Acknowledgments The authors would like to thank D. Frierson, S. Griffies, S. Malyshev, S. Garner, C. Milly and R. Pacanowski for help and discussions at various stages in the development of this study. Two anonymous reviewers provided insightful comments and suggestions on the manuscript and helped improve its presentation. This work was funded by NOAA and NSF.

\section{References}

Barsugli JJ, Battisti DS (1998) The basic effects of atmosphere-ocean thermal coupling on midlatitude variability. J Atmos Sci 55:477493

Bellucci A, Gualdi S, Scoccimarro E, Navarra A (2008) NAO-ocean circulation interactions in a coupled general circulation model. Clim Dyn 31:759-777

Bjerknes J (1964) Atlantic air-sea interaction. Adv Geophys 10:1-82 Cassou C, Deser C, Alexander MA (2007) Investigating the impact of reemerging sea surface temperature anomalies on the winter 
atmospheric circulation over the North Atlantic. J Clim 20:35103526

Colin de Verdière A, Huck T (1999) Baroclinic instability: an oceanic wavemaker for interdecadal variability. J Phys Oceanogr 29:893-910

Czaja A, Marshall J (2001) Observations of atmosphere-ocean coupling in the North Atlantic. Q J R Meteor Soc 127:18931916

Czaja A, Robertson A, Huck T (2003) The role of Atlantic oceanatmosphere coupling in affecting North Atlantic Oscillation variability. In: The North Atlantic oscillation: climatic significance and environmental impact. Geophysical Monograph No. 134, pp 147-172. American Geophysical Union

Dai A, Hu A, Meehl GA, Washington WM, Strand WG (2005) Atlantic thermohaline circulation in a coupled general circulation model: unforced variations versus forced changes. J Clim 18:3270-3293

Danabasoglu G (2008) On multi-decadal variability of the Atlantic meridional overturning circulation in the Community Climate System Model version 3 (CCSM3). J Clim 21:5524-5544

Delworth T, Broccoli A, Rosati A, Stouffer R, Balaji V, Beesley J, Cooke W, Dixon K, Dunne J, Dunne K, Durachta J, Findell K, Ginoux P, Gnanadesikan A, Gordon C, Griffies S, Gudegel R, Harrison M, Held I, Hemler R, Horowitz L, Klein S, Knutson T, Kushner P, Langenhorst A, Lee H-C, Lin S, Lu L, Malyshev S, Milly P, Ramaswamy V, Russel J, Schwarxkopf M, Shevliakova E, Sirutis J, Spelman M, Stern W, Winton M, Wittenberg A, Wyman B, Zeng F, Zhang R (2006) GFDL's CM2 global coupled climate models. Part I: formulation and simulation characteristics. J Clim 19:643-674

Delworth TL, Greatbatch RJ (2000) Multidecadal thermohaline circulation variability driven by atmospheric surface flux forcing. J Clim 13:1481-1495

Delworth TL, Manabe S, Stouffer RJ (1993) Interdecadal variations of the thermohaline circulation in a coupled ocean-atmosphere model. J Clim 6:1993-2011

Delworth TL, Mann M (2000) Observed and simulated multidecadal variability in the Northern Hemisphere. Clim Dyn 16:661676

Deser C, Blackmon M (1993) Surface climate variations over the North Atlantic ocean during winter: 1900-1989. J Clim 6:17431753

Deser C, Thomas R, Peng S (2007) The transient atmospheric circulation response to North Atlantic SST and sea ice anomalies. J Clim 20:4751-4767

Dong B, Sutton RT (2005) Mechanism of interdecadal thermohaline circulation variability in a coupled ocean-atmosphere GCM. J Clim 18:1117-1135

Farneti R, Vallis GK (2009) An intermediate complexity climate model (ICCMp1) based on the GFDL Flexible Modelling System. Geosci Model Dev 2:73-88

Ferreira D, Frankignoul C (2005) The transient atmospheric response to midlatitude SST anomalies. J Clim 18:1049-1067

Frierson DMW (2007) The dynamics of idealized convection schemes and their effect on the zonally averaged tropical circulation. J Atmos Sci 64:1959-1976

Frierson DMW, Held IM, Zurita-Gotor P (2006) A gray-radiation aquaplanet moist GCM. Part I: static stability and eddy scales. J Atmos Sci 63:2548-2566

Frierson DMW, Held IM, Zurita-Gotor P (2007) A gray-radiation aquaplanet moist GCM. Part II: Energy transports in altered climates. J Atmos Sci 64(5):1680-1693

Griffies SM, Gnanadesikan A, Dixon KW, Dunne J, Gerdes A, Harrison MJ, Rosati A, Russel J, Samuels BL, Spelman MJ,
Winton M, Zhang R (2005) Formulation of an ocean model for global climate simulations. Ocean Sci 1:45-79

Griffies SM, Tziperman E (1995) A linear thermohaline oscillator driven by stochastic atmospheric forcing. J Clim 8:2440-2453

Hasselmann K (1976) Stochastic climate models. Part I: theory. Tellus 28:473-485

Hogg A, Dewar W, Killworth P, Blundell J (2006) Decadal variability of the midlatitude climate system driven by the ocean circulation. J Clim 19:1149-1166

Huck T, Vallis G (2001) Linear stability analysis of the threedimensional thermally-driven ocean circulation: application to interdecadal oscillations. Tellus 53A:526-545

Hunke EC, Dukowicz JK (1997) An elastic-viscous-plastic model for sea ice dynamics. J Phys Oceanogr 27:1849-1867

Kamenkovich IV, Sokolov AP, Stone PH (2002) An efficient climate model with a 3D ocean and a statistical-dynamical atmosphere. Clim Dyn 19: 585-598

Kravtsov S, Ghil M (2004) Interdecadal variability in a hybrid coupled ocean-atmosphere-sea-ice model. J Phys Oceanogr 34:1756-1775

Kushnir Y (1994) Interdecadal variations in the North Atlantic sea surface temperature and associated atmospheric conditions. J Clim 7:141-157

Latif M, Barnett TP (1994) Causes of decadal climate variability over the North Pacific and North America. Science 266:634-637

Marshall J, Johnson H, Goodman J (2001) A study of the interaction of the North Atlantic Oscillation with ocean circulation. J Clim 14:1399-1421

Montoya M, Griesel A, Levermann A, Mignot J, Hofmann M, Ganopolsky A, Rahmstorf S (2005) The earth system model of intermediate complexity CLIMBER-3 $\alpha$. Part I: description and performance for present-day conditions. Clim Dyn 25:237-263

Msadek R, Frankignoul C (2008) Atlantic multidecadal oceanic variability and its influence on the atmosphere in a climate model. Clim Dyn. doi:10.1007/s00382-008-0452-0

Preisendorfer RW, Mobley CD (1988) Principal component analysis in meteorology and oceanography. Elsevier, Amsterdam, p 425

Saravanan R, Danabasoglu G, Doney S, McWilliams J (2000) Decadal variability and predictability in the midlatitude oceanatmosphere system. J Clim 15:1073-1097

Saravanan R, McWilliams JC (1995) Multiple equilibria, natural variability, and climate transitions in an idealized oceanatmsophere model. J Clim 8:2296-2323

Saravanan R, McWilliams JC (1998) Advective ocean-atmosphere interaction: an analytical stochastic model with implications for decadal variability. J Clim 11:165-188

Sutton RT, Allen MR (1997) Decadal predictability of North Atlantic sea surface temperature and climate. Nature 388:563-565

Timmermann A, Latif M, Voss R, Grotzner A (1998) Northern hemispheric interdecadal variability: a coupled air-sea mode. J Clim 11:1906-1931

Vallis GK (2006) Atmospheric and oceanic fluid dynamics: fundamentals and large-scale circulation. Cambridge University Press, Cambridge, p 745

Vallis GK, Gerber EP (2008) Local and hemispheric dynamics of the North Atlantic oscillation, annular patterns and the zonal index. Dyn Atmos Oceans 44:184-212

von Storch H, Zwiers FW (2001) Statistical analysis in climate research. Cambridge Univesity Press, Cambridge, p 484

Winton M (2000) A reformulated three-layer sea ice model. J Atmos Ocean Tech 17:525-531

Zhu X, Jungclaus J (2008) Interdecadal variability of the meridional overturning circulation as an ocean internal mode. Clim Dyn 31(6):731-741 